\title{
A multi-data based quantitative model for the neurovascular coupling in the brain.
}

Sebastian Sten ${ }^{1,2}$, Henrik Podéus ${ }^{1}$, Nicolas Sundqvist ${ }^{3}$, Fredrik Elinder ${ }^{4}$, Maria Engström ${ }^{1} 2^{*}$, Gunnar Cedersund ${ }^{2,3^{*}}$

* These authors contributed equally to this work.

${ }^{1}$ Department of Health, Medicine and Caring Sciences, Linköping University, Linköping, Sweden

${ }^{2}$ Center for Medical Image Science and Visualization (CMIV), Linköping University, Linköping, Sweden

${ }^{3}$ Department of Biomedical Engineering, Linköping University, Linköping, Sweden

${ }^{4}$ Department of Biomedical and Clinical Sciences, Linköping University, Linköping, Sweden

\section{Abstract}

The neurovascular coupling (NVC) forms the foundation for functional imaging techniques of the brain, since NVC connects neural activity with observable hemodynamic changes. Many aspects of the NVC have been studied both experimentally and with mathematical models: various combinations of blood volume and flow, electrical activity, oxygen saturation measures, blood oxygenation level-dependent (BOLD) response, and optogenetics have been measured and modeled in rodents, primates, or humans. We now present a first inter-connected mathematical model that describes all such data types simultaneously. The model can predict independent validation data not used for training. Using simulations, we show for example how complex bimodal behaviors appear upon stimulation. These simulations thus demonstrate how our new quantitative model, incorporating most of the core aspects of the NVC, can be used to mechanistically explain each of its constituent datasets. 


\section{Introduction}

The adult brain is an energy-costly organ that consumes $\sim 20 \%$ of a human's total energy consumption [1]. Despite this high energy demand, the brain constitutes only $\sim 2 \%$ of the total bodyweight of an average adult [1]. To meet the energy demand, the brain requires a continuous supply of metabolic substrates such as glucose and oxygen [2]. This continuous supply of substrates is met by diffusion and receptor-mediated transport from the blood to cerebral tissue through the capillaries. Thus, as first reported by Roy and Sherrington in 1890 [3], there is a tight temporal and spatial connection between brain activity and hemodynamics. This connection is commonly referred to as the neurovascular coupling (NVC). The NVC describes the coupling between neural cells and blood vessels and involves changes in the cerebral blood flow (CBF), cerebral blood volume (CBV), and the cerebral metabolic rate of oxygen $\left(\mathrm{CMRO}_{2}\right)$. The NVC is mediated by the synaptic activity of neurons brain activity - that trigger the release of different vasoactive molecules [4-8] (see Fig. 1A for an overview of central signaling pathways). These molecules induce changes in CBF and CBV by acting on vascular smooth muscle cells and pericytes, which enwrap arterioles and capillaries, respectively. NVC is important because it underpins neuroimaging techniques that are based on the usage of hemodynamic changes as a proxy for neural activity $[9,10]$, such as the widely used functional magnetic resonance imaging (fMRI) [11]. However, the interpretation of fMRI data is usually done in a statistical way that ignores the understanding of the underlying biological mechanisms. This is because a quantitative and useful mechanistically resolved model for the NVC remains to be determined.

Previous studies of the NVC have employed a variety of different measurement strategies, each with their own possibilities, strengths, and drawbacks [12]. One technique is the usage of in vitro experiments where brain slices are studied to elucidate intracellular signaling intermediaries in different cell types $([5,6]$, and references therein). While this technique allows for detailed probing of intracellular mechanisms, it does not allow for probing the interaction between these cell types and blood flow. Another technique is invasive in vivo animal experiments. Such experiments are predominantly carried out during anesthesia in rodents $[13,14]$. While such experiments have limited capability to measure intracellular signaling intermediaries, they allow for examinations of the crosstalk between blood supply and brain activity, and for measurements of a wide variety of entities, such as CBF, CBV, vessel diameter, hemoglobin and oxygen saturation changes, as well as electrical activity such as local field potential (LFP) [13-15]. A key possibility in such animal experiments is the usage of optogenetic techniques [16], which allows for the activation of specific neuronal cell types. While most such animal experiments are done in rodents, Logothetis et al., have done important studies in higher primates $[9,10,17,18]$. In two of these studies, they measured LFP and fMRI simultaneously $[9,10]$. Most of these animal studies are performed with the use of anesthetics, which affects neuronal excitability, cerebral metabolism, vascular reactivity amongst other physiological processes [19-22], which complicate the interpretation of such studies. Finally, a variety of non-invasive techniques are also available in humans, most of which use magnetic resonance imaging (MRI). Using MRI, one can measure blood oxygen level-dependent (BOLD) responses to different stimuli, and also measure CBF and CBV. In summary, components of the NVC can be measured by different techniques in different experimental systems, but no system alone allows for simultaneous measurement of all these components (see Fig. 1B for a condensed overview), and especially not in humans.

The NVC is highly conserved across different species, but despite this, there does not exist a systematic overview or synthesis of different data sources. The NVC is often non-invasively characterized using the canonical hemodynamic response function (HRF) extracted from BOLD-fMRI. The HRF consists of two or three different phases: a debated initial dip, the main response, and a post-peak undershoot. The shape of the HRF is consistent across species, such as rodents, primates, and humans. Furthermore, the timing is preserved across these species: in response to a sub-second stimulus, the peak of the main response lies at approximately three to six seconds after a stimulus, and the entire HRF usually lasts $15-20$ seconds [23-25]. Because of this high conservation across species, it is relevant to consider data from different sources together, since they all provide different pieces to the same puzzle. One way to lay such a systems-level puzzle considering data from different experimental systems is to use mathematical modeling.

Previously, various aspects of the NVC have been modeled. In the standard interpretation of BOLDfMRI, activity is equated by correlation with the canonical HRF ([26] and references therein). This approach can provide an estimate of where, in the brain, neuronal activity is present, but this approach 
ignores the neural and vascular mechanisms involved in the NVC. The first serious attempt at describing these mechanisms was made with the 'Balloon' model [27-29]. In this model, the volume of the venules is described by similar mechanisms as those governing the expansion of a balloon. While the Balloon model describes a reasonable series of steps involving neuronal activity, CBF, CBV, $\mathrm{CMRO}_{2}$, and the BOLD signal, the equations describing each of these individual steps are purely phenomenological. The most mechanistically correct way to describe blood flow in vessels is to solve the Navier-Stokes equations [30,31]. In practice, a simplified approach called Windkessel models using lumped parameter models and ordinary differential equations is typically sufficient to provide a sufficient description of the interplay between CBF and CBV [32, 33]. There are a few models that describe the interplay between Windkessel dynamics in arterioles and venules, and their interplay with the BOLD signal [28, 34-37]. However, no such model describes the crosstalk between these processes and the intracellular signaling resulting in the release of vasoactive substances (Fig. 1A). Nevertheless, there are a few models that describe these intracellular signaling cascades and their impact on the blood flow regulation, even though these models use a single blood compartment [3840]. A summary of previous NVC models is found in Fig. 1D. One of the more advanced models describing intracellular pathways is based on optogenetics data, which has unraveled the crosstalk between pyramidal cells and two different types of interneurons [41]. However, no model of the NVC describes such intracellular pathways and all of the above described data available in rodents, primates, and humans.

Herein, we present a first mathematical model that covers multiple aspects of the NVC into one complete model (Fig. 1C \& 2A-C). We have connected a mechanistic NVC model for the control of arterioles (presented in [41]) with a Windkessel model of blood flow, pressure, volume, and

hemoglobin content in arterioles, capillaries, and venules (presented by Barrett et al. [34, 42, 43]), which finally is combined into a complete description of the BOLD signal using the model by Griffeth et al. [44]. Our approach is the first model that is able to describe previously published data from different species (humans, mice, and macaques), consisting of multiple different measurement variables (diameter change of the vessels, different measures of CBV, CBF, transient hemoglobin changes, BOLD responses, and LFP) gathered using both optogenetic and sensory perturbations. Furthermore, the model can successfully predict independent experimental data used for validation. Using the model, we provide mechanistic insights explaining the dynamic behavior seen in each dataset by illustrating how this model can be used to unravel the mechanisms of complicated dynamics observed in different available data sources. To the best of our knowledge, this model constitutes the most complete mathematical description of the NVC to date. 
A

A

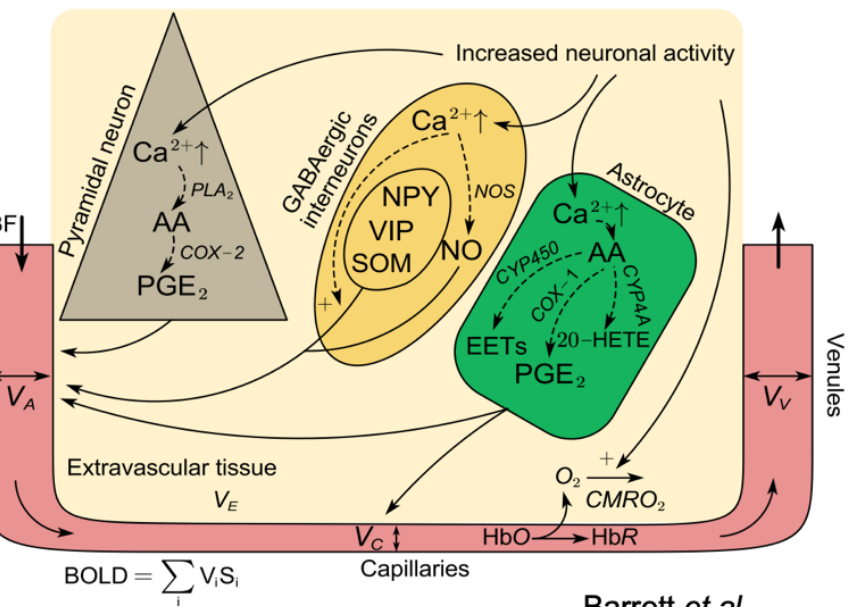

Barrett et al.,

C

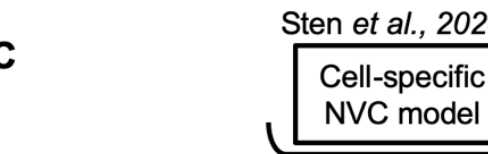

2012, 2013, 2015

Hemodynamics model
B

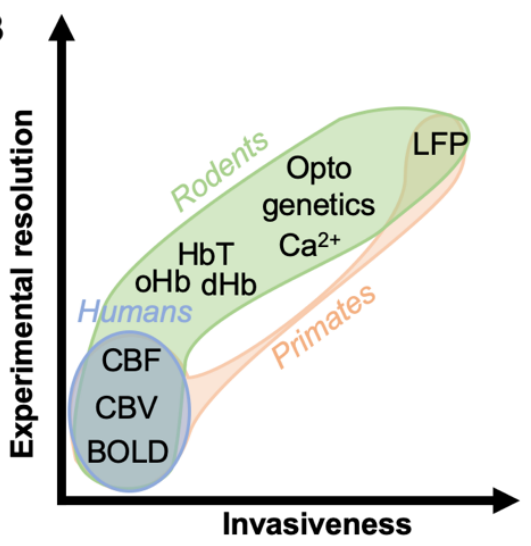

Griffeth et al., 2011

BOLD model

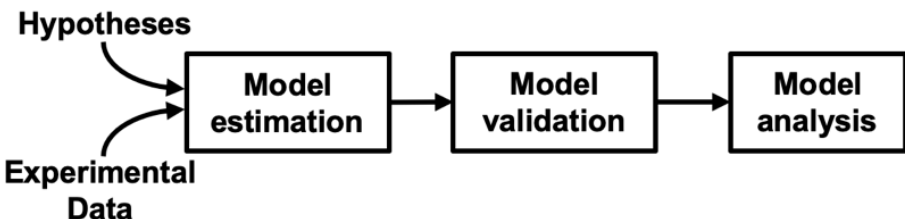

Data

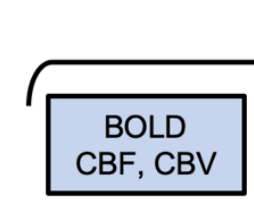

Huber et al., 2014 Humans

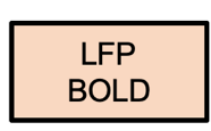

Shmuel et al., 2006

Primates

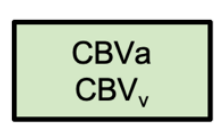

Drew et al., 2011 Rodents

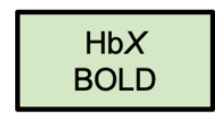

Desjardins et al., 2019 Rodents

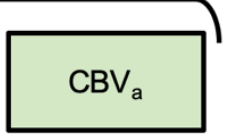

Uhlirova et al., 2016

Rodents

D

\begin{tabular}{|c|c|c|c|c|c|c|c|}
\hline Model & $\begin{array}{l}\text { Lundengård et } \\
\text { al., 2016; Sten et } \\
\text { al., 2017, 2020 }\end{array}$ & $\begin{array}{l}\text { Yücel } \\
\text { et al., } \\
2009\end{array}$ & $\begin{array}{l}\text { Mathias } \\
\text { et al., } \\
2016\end{array}$ & $\begin{array}{l}\text { Buxton et al.,1998, } \\
\text { 2004; Friston et al., } \\
\text { 2000; Havlicek et al., } \\
2015,2017\end{array}$ & $\begin{array}{l}\text { Barrett et } \\
\text { al., 2012, } \\
\text { 2013, 2015 }\end{array}$ & $\begin{array}{l}\text { Huppert } \\
\text { et al., } \\
\text { 2007, 2009 }\end{array}$ & $\begin{array}{l}\text { This } \\
\text { work }\end{array}$ \\
\hline $\begin{array}{c}\text { Intracellular } \\
\text { mechanisms }\end{array}$ & Yes & Yes & Yes & No & No & No & Yes \\
\hline $\begin{array}{c}\text { All vascular } \\
\text { compartments }\end{array}$ & No & No & No & No & Yes & Yes & Yes \\
\hline $\begin{array}{c}\text { Hemoglobin } \\
\text { dynamics }\end{array}$ & No & Partly & Partly & Partly & Yes & Yes & Yes \\
\hline $\begin{array}{c}\text { Full BOLD } \\
\text { description }\end{array}$ & Partly & Partly & Partly & Partly & No & Yes & Yes \\
\hline
\end{tabular}

Figure 1 Overview of the presented study. A: Simplified schematic illustration of the cellular pathways underlying the NVC. Neuronal signaling activates GABAergic interneurons, pyramidal neurons, and astrocytes by stimulating the influx of $\mathrm{Ca}^{2+}$, and increases the cerebral metabolic rate of oxygen $\left(\mathrm{CMRO}_{2}\right)$. $\mathrm{Ca}^{2+}$ facilitates signaling pathways in the different cells. In GABAergic interneurons, $\mathrm{Ca}^{2+}$ promotes nitric oxide (NO) through the upregulation of nitric oxide synthase (NOS). Furthermore, $\mathrm{Ca}^{2+}$ also facilitates the release of different neuropeptides: neuropeptide Y (NPY), vasoactive intestinal peptide (VIP), and somatostatin (SOM). In pyramidal neurons, $\mathrm{Ca}^{2+}$ promotes the synthesis of the arachidonic acid $(A A)$ via phospholipase $A 2\left(P L A_{2}\right)$, which in turn is metabolized to prostaglandin E2 ( $\left.\mathrm{PGE}_{2}\right)$ via cyclooxygenase-2 (COX-2). In astrocytes, arachidonic acid is metabolized into three different vasoactive messengers: PGE 2 via cyclooxygenase-1 (COX-1),

epoxyeicosatrienoic acid (EET) via cytochrome P450 (CYP) epoxygenase, and 20-Hydroxyeicosatetraenoic acid (20-HETE) via CYP4A. Together these vasoactive messengers act on arterioles and capillaries to modulate the vessel diameters, inducing changes in blood flow and volume. These flow and volume changes are propagated throughout the different vessels, giving rise to changes in oxygen saturation, hemoglobin concentrations, and finally, the BOLD signal. B: Overview of commonly used experimental techniques, depicted along two axes: the spatial and temporal resolution of what is measured, and how invasive the technique is. C: Overview of the 
bioRxiv preprint doi: https://doi.org/10.1101/2021.03.25.437053; this version posted March 26,2021 . The copyright holder for this preprint (which was not certified by peer review) is the author/funder, who has granted bioRxiv a license to display the preprint in perpetuity. It is made available under aCC-BY-NC 4.0 International license.

study. Already published experimental data from different species and/or experimental techniques are combined with the development of a mathematical model, which builds upon three already published models. D:

Comparison of different published models describing the neurovascular coupling (NVC) with regards to different mechanisms. The notations used in the table is: 'Yes' if the model features the stated mechanism, 'Partly' if the model has a description that is not fully satisfying, and 'No' if there is no description of the mechanism. As seen, no model exists that can describe every mechanism. HbX: hemoglobin measures; $\mathrm{CBV}_{\mathrm{a}}, \mathrm{CBV}_{\mathrm{v}}$ : compartment specific measures of CBV. 


\section{Methods}

\subsection{Model Formulation}

The model is formulated using a mixture of ordinary differential equations (ODEs) and differential algebraic equations (DAEs). The general notation of such a model is as follows:

$$
\begin{gathered}
F(\dot{x}, x, z, \theta, u, t)=0 \\
F\left(\dot{x}\left(t_{0}, \theta\right), x\left(t_{0}, \theta\right), z\left(t_{0}, \theta\right), t_{0}\right)=0 \\
\widehat{y}=g(x, z, \theta, u, t)
\end{gathered}
$$

in which $x$ is the vector of state variables for which derivatives are present; $z$ is the vector of state variables with no specified derivatives; $\dot{x}$ represent the derivatives of the states with respect to time $t$; $F$ and $g$ are non-linear smooth functions; $\theta$ is the vector of unknown constant parameters; where $u$ is the input signal corresponding to experimental data; where eq. $1 \mathrm{~b}$ is the initial value solution, solving the values of the states at the initial time point $t=t_{0}$, which are dependent on the parameters $\theta$; and where $\hat{y}$ are the simulated model outputs corresponding to the measured experimental signals. Note that $x, z, \dot{x}, u$ and $\hat{y}$ depend on $t$, but that the notation is dropped unless explicitly needed.

\subsection{Model structure}

The model interaction graph is depicted in Figure 2. The interaction graph describes all of the reactions and interactions of the model. In practice, the presented model is the combination of multiple previous studies, where Section 2.2.1-2.2.3 builds upon the work of [41], 2.2.4-2.2.5 corresponds to the work of Barrett et al. [34, 42, 43], and finally, 2.2.6 corresponds to the work of Griffeth et al. [44]. The stimulus $u$ is given by the following equation:

$$
u_{i}= \begin{cases}1 & t_{o n} \leqslant t \leqslant t_{o f f} \\ 0 & \text { otherwise }\end{cases}
$$

where $t_{\mathrm{on}}, t_{\mathrm{off}}$ are the times when the signal goes on and off, respectively. 


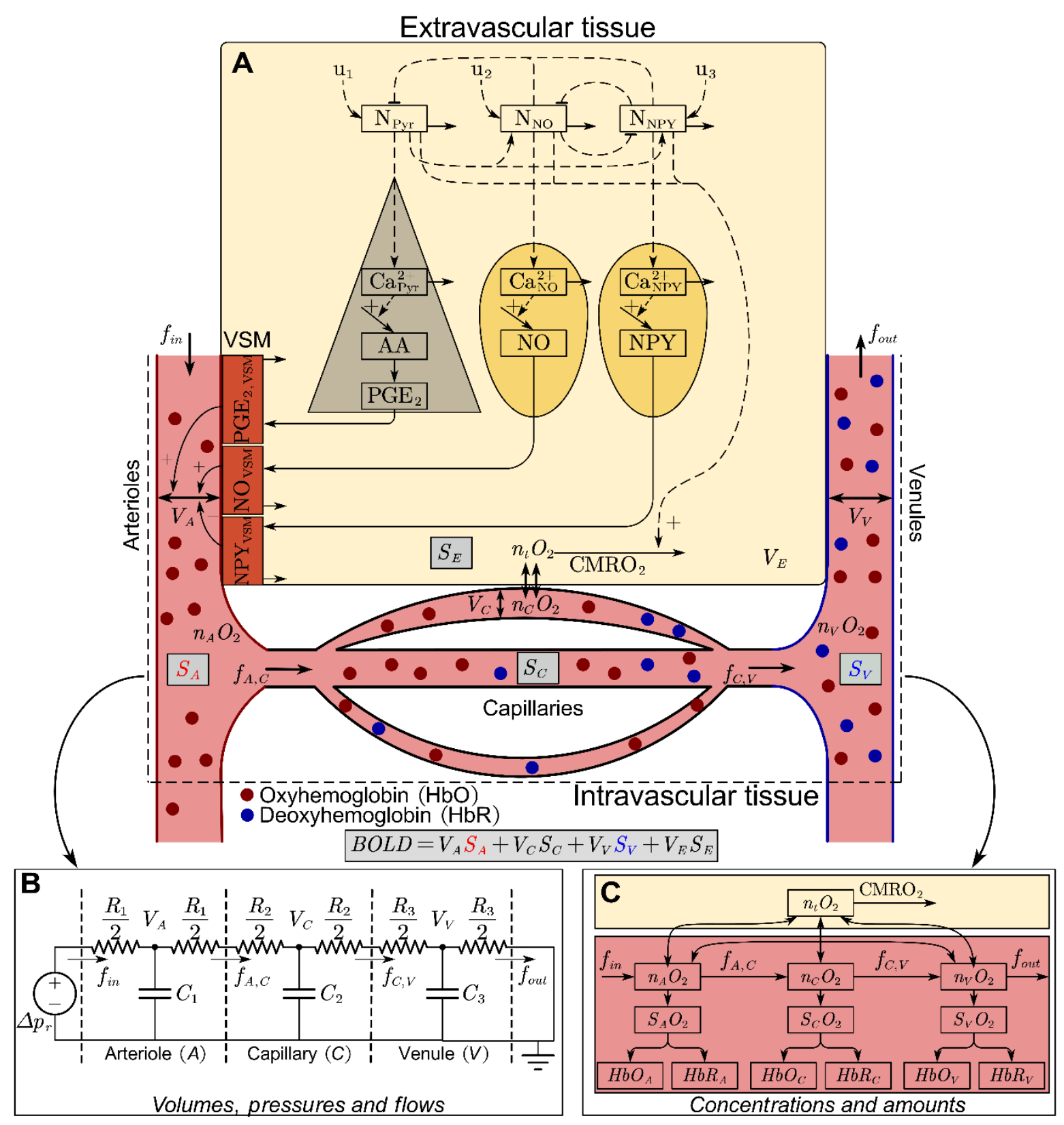

Figure 2 Interaction graph of the model. A: A simplified overview of the model described in [41]. Two distinct types of cells are described: pyramidal neurons (grey triangle, left) and GABAergic interneurons (divided into NO- and NPY-expressing neurons; yellow oval shapes, right). These neurons are connected in a simple relationship, with pyramidal neurons exciting GABAergic neurons, which in turn inhibit the surrounding cells. Activation of a neuron causes an influx of $\mathrm{Ca}^{2+}$ ions. In pyramidal neurons, $\mathrm{Ca}^{2+}$ activates phospholipases, which converts phospholipids into arachidonic acid (AA). AA is further metabolized into prostaglandin E2 (PGE2). In NOexpressing interneurons, $\mathrm{Ca}^{2+}$ activates nitric oxide synthase, which triggers the production and release of nitric oxide (NO). In other subtypes of GABAergic interneurons, vesicle-bound vasoactive peptides such as neuropeptide Y (NPY) are expressed. The release of these peptides is facilitated by $\mathrm{Ca}^{2+}$. Vascular smooth muscle (VSM) cells (brown rectangles) enwrap arterioles (left side of the vascular tree) and regulates the arteriolar diameter. $\mathrm{PGE}_{2}$ promotes arteriolar dilation by activation of the prostaglandin $\mathrm{EP}_{4}$ receptor located on the VSM. NO diffuse freely over cellular membranes, and acts to increase the production of cyclic guanosine monophosphate, which in turn promotes arteriolar dilation. Lastly, NPY activates the G-protein coupled NPY Y1 receptor expressed on VSM cells, promoting arteriolar constriction. These three effects on the VSM control the arteriolar diameter and thereby also the volume and flow of blood through the arterioles. These volume and flow changes are propagated through the capillaries and venules. B: Three-compartment vascular model of blood volumes, pressures, and flows corresponding to an analog electrical circuit, as described in [34]. The blood pressure drop corresponds to a voltage drop, the blood flow to electric current, and the blood volume to electric charge stored in the capacitors. The vessel compliance plays the role of capacitance, and the vessel resistance is analogous to electric resistance. The blood pressure difference maintained by the circulatory system corresponds to the electromotive force. 
C: Oxygen transport model, as described in [42]. The diagram depicts amount of oxygen $\left(\mathrm{n}_{\mathrm{i}} \mathrm{O}_{2}\right)$, oxygen saturation $\left(\mathrm{S}_{\mathrm{i}} \mathrm{O}_{2}\right)$, oxygenated hemoglobin $\left(\mathrm{HbO}_{\mathrm{i}}\right)$ and deoxygenated hemoglobin $\left(\mathrm{HbR}_{\mathrm{i}}\right)$, for each respective compartment $(\mathrm{A}=$ arteriole/arterial, $\mathrm{C}=$ capillary, $\mathrm{V}=$ venule/venous, $\mathrm{t}=$ tissue $)$. Oxygen in tissue can be metabolized, indicated by the cerebral metabolism of $\mathrm{O}_{2}\left(\mathrm{CMRO}_{2}\right)$ arrow leaving the state. All of these different models in unison affect the blood oxygenation and blood volume in each respective compartment, which in turn determines the specific compartments contribution $\left(\mathrm{S}_{\mathrm{i}}\right)$ to the BOLD-fMRI signal (grey boxes).

\subsubsection{Presynaptic activity and calcium influx}

Glutamate and GABA are released from different types of neurons upon membrane depolarization and bind to specific ion channel-coupled receptors located in the neuronal plasma membrane. Glutamate, an excitatory neurotransmitter, binds to $\alpha$-amino-3-hydroxy-5-methyl-4-isoxazolepropionic acid (AMPA) and $N$-methyl-D-aspartate (NMDA) receptors. Activation of these ion channel-coupled receptors triggers ion-conducting pores to open, triggering an influx of $\mathrm{Na}^{+}$and $\mathrm{Ca}^{2+}$ ions which cause a depolarization of the neuron. Depolarization opens voltage-gated calcium channels, allowing a further influx of $\mathrm{Ca}^{2+}$ ions. In contrast, $\gamma$-aminobutyric acid (GABA) acts to prevent depolarization of neurons as it binds to the ion channel-coupled $\mathrm{GABA}_{A}$ or $\mathrm{GABA}_{B}$ receptor, which opens $\mathrm{Cl}^{-}$ionconducting pores. Pyramidal neurons are glutamatergic, meaning that glutamate is released upon depolarization, and acts on e.g., astrocytes and interneurons. GABAergic interneurons release GABA upon depolarization and target pyramidal neurons or other interneurons. This forms a simple relationship, where interneurons regulate the activity of pyramidal neurons. In the model, we represent these interplays between pyramidal and inhibitory interneuron activity and their combined effect on respective neuronal $\mathrm{Ca}^{2+}$ levels as

$$
\begin{gathered}
\frac{d \mathrm{~N}_{\mathrm{NO}}}{d t}=k_{\mathrm{u}, \mathrm{NO}} u_{1}+k_{\mathrm{PF} 1} E_{\mathrm{Pyr}}-k_{\mathrm{IN} 1} E_{\mathrm{NPY}}-\sin k_{\mathrm{N}, \mathrm{NO}} \mathrm{N}_{\mathrm{NO}} \\
\frac{d \mathrm{~N}_{\mathrm{NPY}}}{d t}=k_{\mathrm{u}, \mathrm{NPY}} u_{2}+k_{\mathrm{PF} 2} E_{\mathrm{Pyr}}-k_{\mathrm{IN} 2} E_{\mathrm{NO}}-\sin k_{\mathrm{N}, \mathrm{NPY}} \mathrm{N}_{\mathrm{NPY}} \\
\frac{d \mathrm{~N}_{\mathrm{Pyr}}}{d t}=k_{\mathrm{u}, \mathrm{Pyr}} u_{3}-k_{\mathrm{INF} 1} \mathrm{~N}_{\mathrm{NO}}-k_{\mathrm{INF} 2} \mathrm{~N}_{\mathrm{NPY}}-\sin k_{\mathrm{N}, \mathrm{Pyr}} \mathrm{N}_{\mathrm{Pyr}} \\
\frac{d\left[\mathrm{Ca}_{i}^{2+}\right]}{d t}=k_{\mathrm{Ca}}\left(1+\mathrm{N}_{i}\right)-\sin k_{\mathrm{Ca}, i}\left[\mathrm{Ca}_{i}^{2+}\right] \\
E_{i}= \begin{cases}N_{i} & N_{i} \geqslant 0 \\
0 & N_{i}<0\end{cases}
\end{gathered}
$$

Where $\mathrm{N}_{\mathrm{NO}}, \mathrm{N}_{\mathrm{NPY}}$, and $\mathrm{N}_{\mathrm{Pyr}}$ describe the neuronal activity of the GABAergic nitric oxide (NO) and neuropeptide $Y$ (NPY) interneurons, and the pyramidal neurons, respectively; where $\mathrm{Ca}_{\mathrm{i}}^{2+}$ represents intracellular calcium in each respective neuron; $k_{\mathrm{u}, \mathrm{i}}$ is the input strength of the stimulation to respective neuron; where $k_{\mathrm{PF} 1}$ and $k_{\mathrm{PF} 2}$ are the kinetic rate parameters governing pyramidal to GABAergic interneuron signaling; where $k_{\mathrm{INF} 1}$ and $k_{\mathrm{INF} 2}$ are the kinetic rate parameters describing the negative feedback from GABAergic interneurons to pyramidal neurons; where $k_{\mathrm{IN} 1}$ and $k_{\mathrm{IN} 2}$ are the kinetic rate parameters describing the negative feedback between GABAergic interneurons; where $\sin k_{\mathrm{N}, \mathrm{i}}$ are kinetic rate parameters governing the degradation of the activity of respective neuron; where $k_{\mathrm{Ca}}$ is the basal inflow rate of $\mathrm{Ca}^{2+}\left(k_{\mathrm{Ca}}=10\right.$ for all three neurons), and finally, where $\sin k_{\mathrm{Ca}, \mathrm{i}}$ is the elimination rate of intracellular $\mathrm{Ca}^{2+}$, and $\mathrm{i}=(\mathrm{Pyr}, \mathrm{NO}, \mathrm{NPY})$ denotes the three different neuronal types.

\subsubsection{Pyramidal neuron signaling}

The rise in intracellular $\mathrm{Ca}^{2+}$ levels in pyramidal neurons activates phospholipases which metabolize, through intermediary enzymatic steps, membrane phospholipids into intracellular arachidonic acid (AA) $[12,45,46]$. This is described by: 


$$
\frac{d[\mathrm{AA}]}{d t}=k_{\mathrm{PL}}\left[\mathrm{Ca}_{\mathrm{Pyr}}^{2+}\right]-\frac{k_{\mathrm{COX}}[\mathrm{AA}]}{K_{M, \mathrm{COX}}+[\mathrm{AA}]}
$$

where $k_{\mathrm{PL}}$ and $k_{\mathrm{COX}}$ are kinetic rate parameters and $K_{M, C O X}$ is a Michaelis constant. In pyramidal neurons, $A A$ is metabolized into prostaglandin $E_{2}\left(P E_{2}\right)$ through a cyclooxygenase-2 (COX-2) and $P G E$ synthase rate-limiting reaction $[45,47] . \mathrm{PGE}_{2}$ evoke vasodilation by through activation of EP2 and EP4 receptors expressed on the surface of vascular smooth muscle cells (VSM) cells [45, 48]. In the model, this mechanism described as

$$
\begin{gathered}
\frac{d\left[\mathrm{PGE}_{2}\right]}{d t}=\frac{k_{\mathrm{COX}}[\mathrm{AA}]}{K_{\mathrm{m}, \mathrm{COX}}+[\mathrm{AA}]}-k_{\mathrm{PGE} 2}\left[\mathrm{PGE}_{2}\right] \\
\frac{d\left[\mathrm{PGE}_{2, \mathrm{vsm}}\right]}{d t}=k_{\mathrm{PGE} 2}\left[\mathrm{PGE}_{2}\right]-\sin k_{\mathrm{PGE} 2}\left[\mathrm{PGE}_{2, \mathrm{vsm}}\right]
\end{gathered}
$$

Where $\mathrm{PGE}_{2, \mathrm{vsm}}$ represents $\mathrm{PGE}_{2}$ acting on the VSM cells, and $k_{\mathrm{PGE} 2}$ and $\sin k_{\mathrm{PGE} 2}$ are kinetic rate parameters.

\subsubsection{GABAergic interneuron signaling}

The rise in intracellular $\mathrm{Ca}^{2+}$ levels in GABAergic interneurons evoke the release of different vasoactive messengers and substances. For instance, NO has previously been shown to be a potent vasodilator at the level of arteries and arterioles in both in vitro and in vivo studies [49-52]. NO is released by specific NO-interneurons through a $\mathrm{Ca}^{2+}$ dependent nitric oxide synthase (NOS) ratelimiting reaction (see [6] and references therein). Here, we represent this process as

$$
\begin{gathered}
\frac{d[\mathrm{NO}]}{d t}=k_{\mathrm{NOS}}\left[\mathrm{Ca}_{\mathrm{NO}}^{2+}\right]-k_{\mathrm{NO}}[\mathrm{NO}] \\
\frac{d\left[\mathrm{NO}_{\mathrm{vsm}}\right]}{d t}=k_{\mathrm{NO}}[\mathrm{NO}]-\sin k_{\mathrm{NO}}\left[\mathrm{NO}_{\mathrm{vsm}}\right]
\end{gathered}
$$

where $\mathrm{NO}_{\mathrm{vsm}}$ represents $\mathrm{NO}$ acting on the VSM cells, and $k_{\mathrm{NOS}}, k_{\mathrm{NO}}$ and $\sin k_{\mathrm{NO}}$ are kinetic rate parameters. Here, $\sin k_{\mathrm{NO}}>1 \mathrm{~s}^{-1}$ following experimental work by [53].

Another potent vasoactive messenger is NPY. NPY is released by specific NPY producing subtypes of GABAergic interneurons and has previously been shown to induce vasoconstriction of vessels in vitro $[49,54]$ and in vivo [55]. NPY binds to the NPY receptor Y1, a $\mathrm{G}_{\alpha i}$-protein coupled receptor expressed on the surface of VSM cells enwrapping arteries and arterioles [56-58]. Activation of this receptor inhibits the synthesis of adenosine monophosphate (cAMP) and increase the intracellular $\mathrm{Ca}^{2+}[59]$, leading to VSM contraction and vasoconstriction. In the model, these mechanisms are represented as:

$$
\begin{gathered}
\frac{d[\mathrm{NPY}]}{d t}=k_{\mathrm{NPY}}\left[\mathrm{Ca}_{\mathrm{NPY}}^{2+}\right]-\frac{V_{\mathrm{max}}[\mathrm{NPY}]}{K_{\mathrm{M}}+[\mathrm{NPY}]} \\
\frac{d\left[\mathrm{NPY}_{\mathrm{vsm}}\right]}{d t}=\frac{V_{\mathrm{max}}[\mathrm{NPY}]}{K_{\mathrm{M}}+[\mathrm{NPY}]}-\sin _{\mathrm{NPY}}\left[\mathrm{NPY}_{\mathrm{vsm}}\right]
\end{gathered}
$$

where NPY ${ }_{\mathrm{vsm}}$ represents NPY acting on the VSM cells, and $k_{\mathrm{NPY}}$ and $\sin _{\mathrm{NPY}}$ are kinetic rate parameters. The conversion between intracellular NPY and NPY in the VSM, NPY ${ }_{\mathrm{vsm}}$, is governed by 
Michaelis-Menten kinetics [60,61], where $K_{\mathrm{M}}$ is the Michaelis constant (the concentration of the substrate at which half max reaction rate is achieved) and $V_{\max }$ is the maximal reaction rate.

The expression of the different vasoactive substances is scaled with a parameter, $k_{y_{i}}, i=$ $\{N O, P G E 2, N P Y\}$, and summarized to a total vascular influence $G$ :

$$
\begin{aligned}
G= & k_{\mathrm{y}, \mathrm{NO}}\left(\left[\mathrm{NO}_{\mathrm{vsm}}\right]-\left[\mathrm{NO}_{\mathrm{vsm}, 0}\right]\right)+k_{\mathrm{y}, \mathrm{PGE} 2}\left(\left[\mathrm{PGE} 2_{\mathrm{vsm}}\right]-\left[\mathrm{PGE} 2_{\mathrm{vsm}, 0}\right]\right) \\
& -k_{\mathrm{y}, \mathrm{NPY}}\left(\left[\mathrm{NPY}_{\mathrm{vsm}}\right]-\left[\mathrm{NPY}_{\mathrm{vsm}, 0}\right]\right)
\end{aligned}
$$

\subsubsection{Cerebrovascular dynamics described by an electrical circuit analog model}

We describe the dynamics of three cerebrovascular compartments, corresponding to arteries/arterioles $(a)$, capillaries $(c)$, and veins/venules $(v)$, respectively. The compartments are represented by an electrical circuit analogy, as originally presented in the work by Barrett et al. [34, 42, 43]. We use the derivative work of [62], and only present the fully derived equations to improve the clarity for the reader. We refer the reader to the original articles for an in-depth description of the equations $[34,42,43,62]$. The following equations describe the volume change for each respective cerebrovascular compartment, $\mathrm{V}_{i}$, with $i=\{a, c, v\}$.

$$
\begin{gathered}
\frac{d \mathrm{~V}_{a}}{d t}=\frac{1}{k_{\mathrm{vis}, a}}\left(\frac{K_{a}-\frac{\mathrm{V}_{a}}{\mathrm{~V}_{a, 0}}}{K_{a}-1}+G-\frac{2}{C_{\mathrm{a}, 0}} \frac{\mathrm{V}_{a}}{\left(R_{1}+R_{2}\right) f_{1}+\left(R_{2}+R_{3}\right) f_{2}+R_{3} f_{3}}\right) \\
\frac{d \mathrm{~V}_{c}}{d t}=\frac{1}{k_{\mathrm{vis}, c}}\left(\frac{K_{c}-\frac{\mathrm{V}_{c}}{\mathrm{~V}_{c, 0}}}{K_{c}-1}-\frac{2}{C_{c, 0}} \frac{\mathrm{V}_{c}}{\left(R_{2}+R_{3}\right) f_{2}+R_{3} f_{3}}\right) \\
\frac{d \mathrm{~V}_{v}}{d t}=\frac{1}{k_{\mathrm{vis}, v}}\left(\frac{K_{v}-\frac{\mathrm{V}_{v}}{\mathrm{~V}_{v, 0}}}{K_{v}-1}-\frac{2}{C_{v, 0}} \frac{\mathrm{V}_{v}}{R_{3} f_{3}}\right)
\end{gathered}
$$

Here, $K$ are stiffness coefficients; $k_{v i s, i}$ are viscoelastic parameters; $C_{i}$ represents the compliance of the vessel; $R$ represents the vessel resistance; $f$ represents the flow of blood between the cerebrovascular compartments; the baseline value is indicated by the subscript 0 , and finally, $G$ is the vasoactive function translating the actions of the vasoactive arms (Eq. 8) into hemodynamic changes. In short, the arterial compartment is actively regulated by the vasoactive arms (Eq. 8 \& 9a), and the evoked hemodynamic changes in the arterial vessels are propagated through capillary and venous compartments.

Using conservation of mass, the rate at which the blood volume change in a compartment is given by the difference between in- and outflow of blood:

$$
\frac{d \mathrm{~V}_{i}}{d t}=f_{i-1}-f_{i}
$$

Restructuring the equation gives the following relationship:

$$
f_{i}=f_{i-1}-\frac{d \mathrm{~V}_{i}}{d t}
$$

Next, the pressure drop over a compartment is given by:

$$
\Delta P_{i}(t)=\frac{1}{2} \sum_{i=1} R_{i}\left(f_{i-1}+f_{i}\right)
$$

Which is subject to the pressure boundary conditions:

$$
\sum_{i=1}^{3} \Delta P_{i}=\Delta P_{r}=1
$$

Using this conditions, we can solve for the inflow of blood into the first compartment $f_{0}$ : 


$$
f_{0}=\frac{2-\left(\left(R_{1}+R_{2}\right) f_{1}+\left(R_{2}+R_{3}\right) f_{2}+R_{3} f_{3}\right)}{R_{1}}
$$

The equations above form a DAE system, comprised of three volumes and four flows. To further simplify, the scales of the variables are normalized to:

$$
\left[\begin{array}{c}
\mathrm{V}_{0}=\sum_{i=a}^{v} \mathrm{~V}_{i, 0}=1 \\
f_{i, 0}=1 \\
R_{0}=\sum_{i=a}^{v} R_{i, 0}=1
\end{array}\right]
$$

Using this, the rest of the physiological terms can be expressed as:

$$
\begin{gathered}
P_{i, 0}=\left\{\begin{array}{c}
\Delta P_{r} \quad \text { if } i=a \\
\Delta P_{r}-f_{i, 0} \sum_{j=1}^{i-1} R_{j, 0} \text { if } i=\{c, v\}
\end{array}\right. \\
C_{i, 0}=\frac{\mathrm{V}_{i, 0}}{P_{i, 0}-\frac{1}{2} R_{i, 0} f_{i, 0}} \\
l_{i}=\left(R_{i, 0} \mathrm{~V}_{i, 0}^{2}\right)^{\frac{1}{3}} \\
R_{i}=\frac{l_{i}^{3}}{\mathrm{~V}_{i}^{2}}
\end{gathered}
$$

where $P$ is pressure at the entry point to each compartment, $C$ is the compliance of the vascular vessel, $L$ is the length of the vascular segment, and $R$ is the vessel resistance to flow.

Given the Equations 9-16, the initial conditions for the cerebrovascular volumes $V_{i}$ and the vascular resistances $R_{i}$ must be specified. We set these initial conditions in accordance to the work of Barrett et al. [34], which based these values on available literature, resulting in $\mathrm{V}_{i, 0}=[0.29,0.44,0.27]$ and $R_{i, 0}=$ $[0.74,0.08,0.18]$.

\subsubsection{Oxygen transportation}

To describe the oxygen transportation through the cerebrovascular system, we describe three vascular compartments and a cerebral-tissue compartment. The amount of oxygen in these compartments is given by:

$$
\begin{gathered}
\frac{d \mathrm{n}_{a} \mathrm{O}_{2}}{d t}=f_{0} c_{O_{2}, i n}-f_{1} c_{O_{2}, a, c}-j_{O_{2}, a}-j_{O_{2}, s} \\
\frac{d \mathrm{n}_{c} \mathrm{O}_{2}}{d t}=f_{1} c_{O_{2}, a, c}-f_{2} c_{O_{2}, c, v}-j_{O_{2}, c} \\
\frac{d \mathrm{n}_{v} \mathrm{O}_{2}}{d t}=f_{2} c_{O_{2}, c, v}-f_{3} c_{O_{2}, o u t}-j_{O_{2}, v}+j_{O_{2}, s} \\
\frac{d \mathrm{n}_{t} \mathrm{O}_{2}}{d t}=\sum_{i} j_{O_{2}, i}-C M R O_{2}
\end{gathered}
$$

where the flow of blood $f$ transports oxygen in and out from each compartment. The permeability in the vessel wall allows for oxygen to diffuse to the tissue compartment, $j_{o_{2}, i}$, where $i=\{a, c, v\}$. An arterio-venous diffusion shunt is described by $j_{O_{2}, s}$, where oxygen moves from the arterial to venous compartment. Oxygen is metabolized in the tissue compartment, $\mathrm{CMRO}_{2}$, which is given by: 


$$
C M R O_{2}=C M R O_{2,0}\left(1+k_{m e t}\left(\mathrm{~N}_{\mathrm{NO}}+\mathrm{N}_{\mathrm{NPY}}+\mathrm{N}_{\mathrm{Pyr}}\right)\right)
$$

where $\mathrm{CMRO}_{2,0}$ is the basal metabolism of oxygen, which is increased by the activity-level of each neuron (see Eq. 3) scaled by the parameter $k_{m e t}$.

The concentration of oxygen, $C_{O_{2}, i}$ is given by the amount of oxygen, $n_{i} O_{2}$, divided by the volume of each compartment, $\mathrm{V}_{i}$, except for the oxygen concentration that enters the arteries, $c_{O_{2}, 0}$, which is assumed to be constant, minus a loss of oxygen to the surrounding tissue $c_{O_{2}, \text { leak }}$.

$$
c_{O_{2}, i, i+1}= \begin{cases}\frac{\mathrm{n}_{i} \mathrm{O}_{2}}{\mathrm{~V}_{i}} & \text { if } i=\{a, c, v, t\} \\ c_{O_{2}, 0}-c_{O_{2}, \text { leak }} & \text { if } i=\text { in }\end{cases}
$$

Where the tissue volume fraction $\mathrm{V}_{t}=34.8$ [42] and $c_{O_{2}, \text { leak }}=0.116 \mathrm{mM}$ (calculated using data from $[42,63])$.

As in the original work [42], by ignoring the minor fraction of oxygen directly dissolved in the blood plasma, blood oxygen concentration can be related to oxygen partial pressures through the oxygenhemoglobin saturation curve:

$$
p_{O_{2}, i, i+1}=p_{50}\left(\frac{c_{O_{2}, \max }}{c_{O_{2}, i, i+1}}-1\right)^{\frac{-1}{h}}
$$

Where $p_{50}=36 \mathrm{mmHg}$ is the oxygen partial pressure at the half-way saturation point, $c_{O_{2}, \max }=$ $9.26 \mathrm{mM}$ is the maximum oxygen concentration in blood, and $h=2.6$ is the Hill exponent. These values are taken from [64].

The oxygen pressure in the tissue, $p_{O_{2}, t}$, is calculated using Henry's law:

$$
p_{O_{2}, t}=\frac{c_{O_{2}, t}}{\sigma_{\text {oxygen }}}
$$

Where $\sigma_{\text {oxygen }}=1.46 \mu \mathrm{M} / \mathrm{mmHg}$ is the coefficient for solubility of oxygen in tissue [65].

The average pressure, $\tilde{p}_{O_{2}, i}$, in a compartment is obtained by averaging over the input and output pressures:

$$
\tilde{p}_{O_{2}, i}=\frac{p_{O_{2}, i-1, i}+p_{O_{2}, i, i+1}}{2}
$$

The diffusion of oxygen between the vessels and the tissue, $j_{O_{2}, i}$, as well as between the arteries and the veins, $j_{O_{2}, s}$, is driven by the difference in partial oxygen pressure:

$$
\begin{aligned}
j_{O_{2}, i}= & g_{i}\left(\tilde{p}_{O_{2}, i}-p_{O_{2}, t}\right), i=\{a, c, v\} \\
& j_{O_{2}, s}=g_{s}\left(\tilde{p}_{O_{2}, a}-\tilde{p}_{O_{2}, v}\right)
\end{aligned}
$$

where $g_{i}, i=\{a, c, v, s\}$ are rate constants.

We employed the identical strategy of Barrett et al. [42] (see supplementary material in [42]), and used the experimental $p_{\mathrm{O}_{2}}$ data from Vovenko et al. [63] to calculate $g_{i}, c_{O_{2}, \text { leak }}$, and $C M R O_{2,0}$. This leaves the parameter $k_{m e t}$ to be estimated where applicable.

The oxygen saturation of blood in the different vascular compartments, $S_{i} O_{2}$, is approximated by dividing the average oxygen concentration of each compartment with $c_{O_{2} \text {, max }}$ :

$$
S_{i} O_{2}=\frac{c_{O_{2}, i-1, i}+c_{O_{2}, i, i+1}}{c_{O_{2}, \max }}, i=\{a, c, v\}
$$

Lastly, using the blood oxygen saturation $S_{i} O_{2}$ and vascular volumes $\left(\mathrm{V}_{i}\right)$, corresponding changes in oxygenated hemoglobin $(\mathrm{HbO})$, deoxyhemoglobin $(\mathrm{HbR})$, and total hemoglobin $(\mathrm{HbT})$ are given by: 


$$
\begin{gathered}
H b T_{\mathrm{i}}=\mathrm{V}_{i} \\
H b O_{i}=\mathrm{V}_{i} S_{i} O_{2} \\
H b R_{i}=\mathrm{V}_{i}\left(1-S_{i} O_{2}\right)
\end{gathered}
$$

\subsubsection{BOLD signal derivation}

Finally, the oxygen saturation of blood in the different vascular compartments, $S_{i} O_{2}$ (Eq. 24) and the vascular volumes, $\mathrm{V}_{i}$ (Eq. 9) are used to calculate the BOLD signal. The following derivation of the BOLD signal equations and parameter values are taken from the work of [44], and we refer the reader to the original article for a detailed view of the equations. The BOLD signal is expressed as a summation of the contributions from each vascular compartment $\{a, c, v\}$ and an extracellular compartment $\{e\}$, symbolizing the tissue:

$$
\begin{gathered}
\mathrm{BOLD}=H \sum_{j} S_{j}, j=\{a, c, v, e\} \\
H=\frac{1}{\mathrm{~V}_{e}+\sum_{k} \varepsilon_{k} \hat{\mathrm{V}}_{k}}, k=\{a, c, v\} \\
\varepsilon_{(a, c, v)}=\frac{S_{(a, c, v), 0}}{S_{e, 0}}=\lambda \frac{e^{-T E R_{2(a, c, v)}^{*}}}{e^{-T E R_{2,0}^{*}}}
\end{gathered}
$$

Where $S_{j}$ is the intrinsic signal from each compartment; $V_{e}=1-\widehat{V}_{k}$ is the extravascular volume; $\widehat{V}_{k}=$ $V_{i v, 0} \sum V_{k}$ is the intravascular volume scaled by the baseline intravascular volume fraction $\left(V_{i v, 0}=0.05\right.$, $[66]) ; \varepsilon_{i}$ is the intrinsic signal ratio of blood and $\lambda$ is the intravascular to extravascular spin density ratio (we assume $\lambda=1.15$ ); $R_{2}^{*}$ is the transverse signal relaxation rate for the four compartments, and TE is the echo time used during image acquisition in each study.

The transverse signal relaxation rate for the extravascular compartment $\left(R_{2 e, 0}^{*}\right)$ was assumed to be $25.1 s^{-1}$ [67], and was calculated for the intravascular compartments by utilizing the following quadratic expression taken from [68]:

$$
R_{2 i, 0}^{*}=A^{*}+C^{*}\left(1-S_{i} O_{2,0}\right)^{2}
$$

where

$$
\begin{aligned}
& A^{*}=14.87 H c t+14.686 \\
& C^{*}=302.06 H c t+41.83
\end{aligned}
$$

These equations are governed by $S_{i} O 2$ (Eq. 24) and the resting hematocrit in blood (Hct; $\mathrm{Hct}_{a, v}=$ $\left.0.44, H_{c t}=0.33[69,70]\right)$.

The respective signal contribution from each compartment is given by:

$$
S_{i}= \begin{cases}\varepsilon_{i} \hat{\mathrm{V}}_{i} e^{-T E \Delta R_{2 i}^{*}} & \text { if } i=\{a, c, v\} \\ \mathrm{V}_{e} e^{-T E \Delta R_{2 e}^{*}} & \text { if } i=e\end{cases}
$$

Where $\Delta R_{2}^{*}$ (i.e., the change in MR signal relaxation rate) is given by the following expressions:

$$
\begin{gathered}
\Delta R_{2 i}^{*}=C^{*}\left(\left(1-S_{i} O_{2}\right)^{2}-\left(1-S_{i} O_{2,0}\right)^{2}\right) \\
\Delta R_{2 e(a, c, v)}^{*}=\left\{\begin{array}{c}
\frac{4 \pi}{3} \Delta \chi H c t \gamma B_{0} \sum_{i=a, v}\left[\hat{\mathrm{V}}_{i}\left(\left|S_{o f f} O_{2}-S_{i} O_{2}\right|\right)-\hat{\mathrm{V}}_{i, 0}\left(\left|S_{o f f} O_{2}-S_{i} O_{2, o}\right|\right)\right] \\
0.04\left(\Delta \chi H c t \gamma B_{0}\right)^{2}\left[\hat{\mathrm{V}}_{c}\left(\left|S_{o f f} O_{2}-S_{c} O_{2}\right|\right)^{2}-\hat{\mathrm{V}}_{c, 0}\left(\left|S_{o f f} O_{2}-S_{c} O_{2, o}\right|\right)^{2}\right]
\end{array}\right.
\end{gathered}
$$

Here, $\Delta \chi=2.64 \times 10^{-7}$ is the susceptibility of fully deoxygenated blood $[71] ; \gamma=2.68 \times 10^{8}$ is the gyromagnetic ratio of protons; $B_{0}$ is the magnetic field strength used during image acquisition, and 
finally, $S_{o f f} O_{2}=0.95$ is the blood saturation which gives no magnetic susceptibility difference between blood and tissue [71].

\subsection{Model evaluation}

\subsubsection{Optimization of parameters}

Once the model structure has been formulated (Fig. 2) and data has been collected (data acquisition described below), the parameters, $\theta$, need to be determined. This is commonly done by evaluating the negative log-likelihood function. Assuming independent, normally distributed additive measurement noise, the likelihood of observing data $D$ given $\theta$ is:

$$
J(\theta)=-\log (L(D \mid \theta))=\frac{1}{2} \sum_{e=1}^{n_{e}} \sum_{o=1}^{n_{o}^{e}} \sum_{s=1}^{n_{s}^{e, o}}\left[\log \left(2 \pi\left(\sigma_{s}^{e, o}\right)^{2}\right)+\left(\frac{y_{s}^{e, o}-\widehat{y}_{s}^{e, o}(\theta)}{\sigma_{s}^{e, o}}\right)^{2}\right]
$$

where $n_{e}$ is the number of experiments $e$; where $n_{o}^{e}$ is the number of observables $o$ per $e$; where $n_{s}^{e, o}$ is the number of samples per $o$ and $e$; where $\sigma_{s}^{e, o}$ is the standard deviation of the data point; where $y_{s}^{e, o}$ is the measured data point; where $\hat{y}(\theta)$ is the corresponding simulated data point. By maximizing $L$, the maximum likelihood estimate of the unknown parameters $\theta$ can be obtained. However, it is more common and more numerically efficient to minimize the equivalent negative log-likelihood function:

If the measurement noise $\sigma_{s}^{e, o}$ is known, $J(\theta)$ share the same optimal parameters with the leastsquares function $J_{l s q}$ :

$$
J_{l s q}(\theta)=\sum_{e=1}^{n_{e}} \sum_{o=1}^{n_{o}^{e}} \sum_{s=1}^{n_{s}^{e, o}}\left(\frac{y_{s}^{e, o}-\widehat{y}_{s}^{e, o}(\theta)}{\sigma_{s}^{e, o}}\right)^{2}
$$

In practice, the parameters are determined by optimizing $J(\theta)$ over $\theta$ by using various optimization algorithms (see Section 2.5 below), and given by:

$$
\widehat{\theta}=\arg \min J(\theta)
$$

where $\hat{\theta}$ are the optimal parameters.

\subsubsection{Simulation uncertainty}

The model uncertainty was estimated using a MCMC-sampling procedure with $10^{5}$ samples (see Section 2.5 for details), generating posterior distributions of the parameters, and collecting all $\chi^{2}$ acceptable parameters encountered. Using the acceptable $\chi^{2}$ parameters, a confidence interval:

$$
C I_{\alpha, d f}=\left(J_{l s q}(\theta) \leq J_{l s q}(\hat{\theta})+\Delta_{\alpha}\left(\chi_{d f}^{2}\right)\right)
$$

was drawn where the threshold $\Delta_{\alpha}\left(\chi_{d f}^{2}\right)$ is the $\alpha$ quantile of the $\chi_{d f}^{2}$ statistic $[72,73]$. We also require that all acceptable parameters should pass a $\chi^{2}$-test.

\subsection{Experimental data}

In this work, experimental data from a total of five different studies have been used, which includes data from rodents, primates, and humans. Below, condensed descriptions of the experimental data are provided, and we kindly refer the reader to the original manuscripts for a complete description of the experimental methods. All data were extracted from figures in the respective original publications using the tool WebPlotDigitizer [74].

\subsubsection{Fractional diameter change of arterioles and venules in mice}

The work of Drew et al. [75] presents experimental data of fractional diameter change in arterioles and venules upon different lengths of a sensory stimulation in mice $(n=21)$, measured using 2-photon 
imaging. The sensory stimulation consisted of trains of air puffs (delivered at $8 \mathrm{~Hz}, 20 \mathrm{~ms}$ duration per puff) directed at the vibrissae, with three different durations: $20 \mathrm{~ms}, 10 \mathrm{~s}$, and $30 \mathrm{~s}$. The mice were awake during the experiment, but were briefly anesthetized before the imaging session using isoflurane ( $2 \%$ in air). We have chosen to consider the period after each puff as a part of the stimulation cycle. This makes the shortest stimulation length of one air puff $125 \mathrm{~ms}$ long. The vascular responses were extracted from figure $2 \mathrm{C}$ in the original publication [75].

\subsubsection{Arteriolar diameter responses for OG and sensory stimuli in mice}

Uhlirova et al. [55] reports arteriolar diameter responses to both $O G$ and sensory stimulations measured using in vivo two-photon imaging, during both awake and under anesthesia conditions in mice. Briefly, four different stimulation protocols were used in their study: three conducted under the administration of the anesthetic $\alpha$-chloralose, and the fourth during awake condition. Below, summarized descriptions of each stimulation protocol are presented, and we refer the reader to the original publication ([55]) and our previous modeling paper [41] for a complete description.

OG stimulation of pyramidal neurons during anesthesia.

Thy1-ChR2-YFP mice ( $\mathrm{n}=2$ subjects), with Channelrhopodsin-2 (ChR2) selectively expressed in layer 5 pyramidal neurons, were imaged (28 measurement locations along 13 arterioles within 40-380 $\mu \mathrm{m}$ range) during $O G$ stimulation (single light pulse lasting 50-80 ms) under both control conditions and under the influence of both the AMPA/kaniate receptor antagonist 6-cyano-7-nitroquinoxaline-2,3dione (CNQX), and the NMDA receptor-selective antagonist D-(-)-2-Amino-5-phosphonopentanoic acid (AP5).

OG stimulation of GABAergic interneurons during anesthesia.

VGAT-ChR2(H134R)-EYFP mice ( $\mathrm{n}=3$ subjects), with ChR2 selectively expressed in GABA interneurons, were imaged (52 measurement locations along 25 arterioles within 50-590 $\mu \mathrm{m}$ range) during OG stimulation (one pair of light pulses separated by $130 \mathrm{~ms}$, for a total of $450 \mathrm{~ms}$ duration) under both a control condition and during the application of the NPY receptor Y1 inhibitor BIBP-3226.

\section{Sensory stimulation during anesthesia.}

Additionally, four wild-type mice underwent imaging (10 measurement locations along 7 arterioles within 130-490 $\mu \mathrm{m}$ range) during sensory stimulation ( $2 \mathrm{~s}$ train of electrical pulses) under both a control condition and during the application of the NPY receptor Y1 inhibitor BIBP-3226.

Sensory and OG stimulation during awake condition.

Lastly, four wild-type and three VGAT-ChR2(H134R)-EYFP awake mice were imaged during sensory stimulation ( $1 \mathrm{~s}$ of air puffs, $100 \mathrm{~ms}$ puffs at $3 \mathrm{~Hz}$ ) and OG stimulation (single light pulse lasting 150$400 \mathrm{~ms})$, respectively.

For the data extracted from this study, standard error of the mean (SEM) in all extracted time series with SEM measurements smaller than mean SEM was set to the mean SEM. This was done to avoid overfitting the model to a few extreme data points.

\subsubsection{Hemoglobin and BOLD responses for $O G$ and sensory stimuli in mice}

Desjardins et al. [76] presents experimental data consisting of optical intrinsic imaging (OIS) of blood oxygenation for both wild-type mice $(n=16)$ and two mice-lines transfected with (ChR2). The mice were kept in an awake state during the data acquisition. In the transfected mice, ChR2 was expressed in pyramidal neurons (Emx1-Cre/Ai32) or in all inhibitory interneurons (VGAT-ChR2(H134R)-EYFP). For the wild-type mice, a sensory stimulus consisting of air puffs $(3-5 \mathrm{~Hz})$ delivered to a whisker pad (duration 2 or $20 \mathrm{~s}$ ) was used to induce hemodynamic responses. The OG-transfected mice were exposed to an OG stimulus consisting of a single $100 \mathrm{~ms}$ light pulse $(473 \mathrm{~nm})$ or a 20 s block of 100 ms light pulses delivered at $1 \mathrm{~Hz}$. The blood oxygenation data were analyzed using the baseline assumption of $60 \mu M \mathrm{HbO}$ and $40 \mu M \mathrm{HbR}$. In addition, some Emx1-Cre/Ai32 mice underwent BOLD fMRI imaging for the $20 \mathrm{~s}$ OG stimulus using gradient-echo echo-planar-imaging (GE-EPI) readout $(\mathrm{TE} / \mathrm{TR}=11-20 \mathrm{~ms} / 1 \mathrm{~s})$. 


\subsubsection{Electrophysiological and BOLD responses in macaque monkeys}

The study by Shmuel et al. [10] reports simultaneously measured electrophysiological responses by invasive recording, and non-invasively measured BOLD responses. These measurements were carried out in the visual cortex of anesthetized macaques at 4.7 T ( $n=7)$. The stimulus paradigm consisted of a $20 \mathrm{~s}$ visual stimuli featuring flickering radial checkers rotating at $60 \% \mathrm{~s}$. A grey background persisted for $5 \mathrm{~s}$ before and $25 \mathrm{~s}$ after the stimuli. If the visual stimuli overlapped with the receptive field of $\mathrm{V} 1$, it induced positive BOLD responses in close vicinity to the electrode. In contrast, if the visual stimuli were presented outside the receptive field of $\mathrm{V} 1$, it induced negative BOLD responses in the same area. The electrophysiological recordings were processed by averaging the fractional change of the power spectrum over the frequency range of $4-3000 \mathrm{~Hz}$, using a temporal resolution of $1 \mathrm{~s}$. BOLD data were acquired using a gradient-echo echo-planar-imaging (GE-EPI) readout $\left(T E=20 \mathrm{~ms}, T R=1 \mathrm{~s}\right.$, in-plane spatial resolution $\left.0.75 \times 0.75 \times 2 \mathrm{~mm}^{3}\right)$. Positive and negative BOLD responses evoked by the two visual stimuli were sampled and averaged over the same voxels located around the electrode. These processed responses were extracted from Figures 1D (BOLD) and 2A (electrophysiological responses) in the original publication [10], respectively. The SEM in all extracted electrophysiological time series with SEM measurements smaller than mean SEM was set to the mean SEM, to avoid overfitting to a few extreme datapoints.

\subsubsection{MR-based hemodynamic measurements in humans}

The study by Huber et al. [77] presents in vivo MR-based measurements of CBF, CBV, and BOLD changes in humans $(n=17)$ evoked using multiple visual stimuli. The study used three different visual stimulation paradigms (alternating $30 \mathrm{~s}$ rest vs. $30 \mathrm{~s}$ stimulation), consisting of two different full-field flickering checkerboards and one small circle flickering checkerboard paradigm. The full-field flickering checkerboard paradigms were used to measure arterial CBV, venous CBV, and total CBV data to both an excitatory and an inhibitory task. The small circle paradigm was used to evoke strong negative BOLD responses, and to concurrently measure both positive and negative BOLD responses, as well as the associated total CBV changes. These data were acquired using a Slice-Saturation SlabInversion Vascular Space Occupancy (SS-SI-VASO) sequence [78] with a multi-gradient echo EPI readout $\left(T E_{1} / T E_{2} / T E_{3} / T I_{1} / T I_{2} / T R=12 / 32 / 52 / 1000 / 2000 / 2500 / 3000 \mathrm{~ms}\right.$, nominal resolution $=\sim 1.3 \mathrm{x}$ $\left.\sim 1.3 \times 1.5 \mathrm{~mm}^{3}\right)$. In addition, CBF changes were acquired from ROls of positive and negative BOLD responses using a pulsed arterial spin label (PASL) sequence in four subjects ( $T E_{1} / T E_{2} / T I_{1} / T I_{2} / T R=$ $8.2 / 19.4 / 700 / 1700 / 3000 \mathrm{~ms}$, nominal resolution $=3 \times 3 \times 3 \mathrm{~mm}^{3}$ ). BOLD and total CBV responses were extracted from Figure. $4 A-B$; arterial $C B V$, venous $C B V$, and total $C B V$ responses were extracted from Figure. 5B-C, and finally, $\mathrm{CBF}$ responses were extracted Figure. $6 \mathrm{C}$ in the original publication.

\subsection{Model implementation}

Implementation and simulation of the model equations (specified in Section 2.2) were carried out using the 'Advanced Multi-language Interface to CVODES and IDAS' (AMICI) toolbox [79, 80]. Parameter estimation for the model evaluation was carried out using the MATLAB implemented 'MEtaheuristics for systems biology and bioinformatics Global Optimization' (MEIGO) [81] toolbox and the enhanced scatter search (eSS) algorithm. In addition, eSS was used in conjunction with two local optimization solvers: dynamic hill climbing [82] and the interior point algorithm included in FMINCON (MATLAB and Optimization Toolbox Release 2017b, The MathWorks, inc., Natick, Massachusetts, United States) paired with the calculation of the objective function gradient using AMICl. For the model uncertainty analysis, we employed the 'Parameter EStimation ToolBox' (PESTO) [83] and the region-based adaptive parallel tempering algorithm [84] to generate the posterior distributions of the parameters. The parameter bounds were $[-4.5,4.5]$ in $\log _{10}$ space unless specified otherwise in Section 2.2.

\subsection{Data and code availability}

The code necessary for reproducing all the figures and results reported herein is provided in the Supplementary material as MATLAB scripts. 


\section{Results}

Below, experimental data gathered from five studies: Drew et al. [75], Desjardins et al. [76], Uhlirova et al. [55] (Section 3.1), Shmuel et al. [10] (Section 3.2), and finally, Huber et al. [77] (Section 3.3) are presented with corresponding model simulations including the uncertainty of the model.

\subsection{NVC measures in awake and anesthetized mice}

\subsubsection{Fractional diameter change of arterioles and venules in awake mice}

The model was trained using experimental data extracted from the study of Drew et al. [75], consisting of arteriolar (Fig. 3A-C, red symbols) and venular (Fig. 3D-F, blue symbols) diameter changes induced by a sensory whisker stimulation for three different durations: $20 \mathrm{~ms}$ (Fig. 3A, D), $10 \mathrm{~s}$ (Fig. $3 \mathrm{~B}, \mathrm{E})$ and $30 \mathrm{~s}$ (Fig. 3C, F). The stimulation consisted of air puffs ( $8 \mathrm{~Hz}, 20 \mathrm{~ms}$ duration), and just as in Barrett et al. [34], we opted to implement this paradigm as one block with the following durations: $125 \mathrm{~ms}, 10 \mathrm{~s}$, and $30 \mathrm{~s}$. In the model, we calculate the diameter change as the square root of the volume change for each respective compartment. The model parameters were fitted to all experimental time series simultaneously (Fig. $3 \mathrm{~A}-\mathrm{F}$, colored symbols), achieving a quantitative acceptable agreement to experimental data (Fig. 3A-F, solid colored lines), evaluated using a $\chi^{2}$-test $\left(U_{l s q}\left(\hat{\theta}_{H 1}\right)=292.59\right.$, cut-off: $\chi^{2}(288$ data points $)=328.58$ for $\left.\alpha=0.05\right)$. The degrees of freedom for the confidence interval was $d f_{H 1}=38$, which is equal to the number of estimated parameters. The estimated model uncertainty is depicted in the form of shaded red and blue areas in Figure 3.

The simulations follow the qualitative behavior of the experimental data for each graph except for Fig. $3 \mathrm{E}$, which is the venular response to a $10 \mathrm{~s}$ stimulation. For that stimulation, the model simulations show a slower rise and decline than the experimental data (Fig. 3E, compare blue line with blue symbols). A more accurate fit can be obtained by allowing the viscoelasticity and stiffness coefficients of the capillary and venous compartments to change between the two short (125 ms \& $10 \mathrm{~s}$ ) and the long (30 s) stimulation (Fig. S1, $\left.J_{l s q}\left(\hat{\theta}_{H 2}\right)=199.56\right)$. This improved agreement indicates that a nonlinear vascular model is needed to fully capture the dynamics seen in this data set. For the other experimental time series, the model simulations closely resemble the experimental data, and the model uncertainty enwraps most of the experimental data points. 
10 s stimulation

30 s stimulation

B

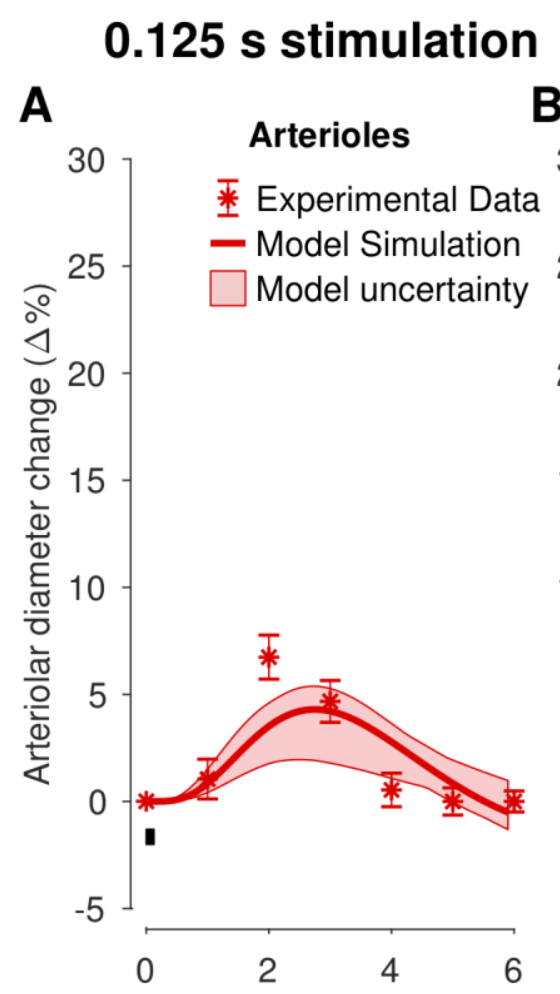

30

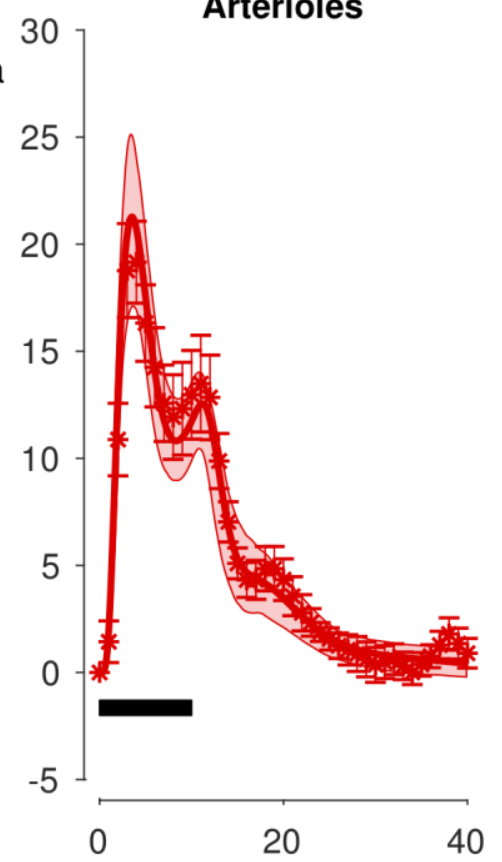

E

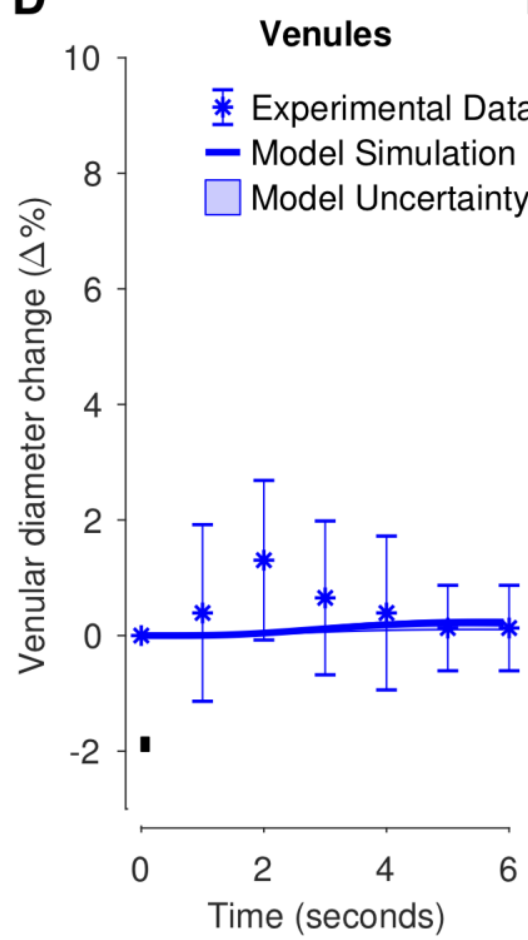

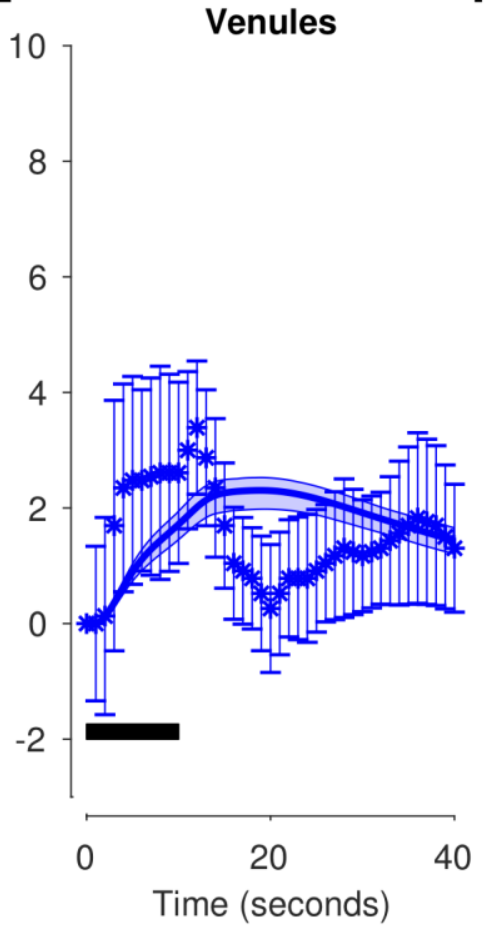

C

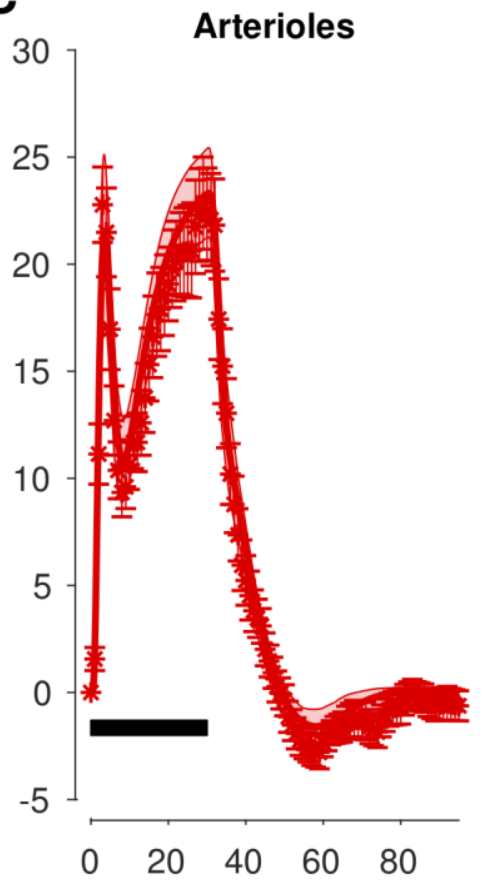

$\mathbf{F}$

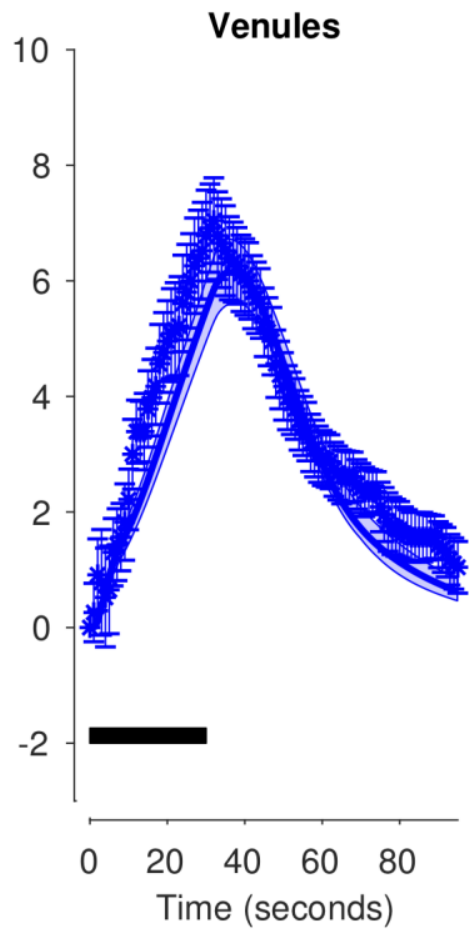

Figure 3 Model estimation to experimental data of arteriolar (A-C) and venular (D-F) diameter changes in awake mice for three different sensory stimulation lengths: $125 \mathrm{~ms}$ (A \& D), $10 \mathrm{~s}$ (B \& E), and $30 \mathrm{~s}$ (C \& F). Experimental data are replotted versions of data presented in Fig. $2 \mathrm{C}$ of the original manuscript by Drew et al. [75]. The stimulation lengths are denoted with the black bar in the bottom left portion of each graph. For each graph: experimental data (colored symbols); the uncertainty of the experimental data is presented as SEM (colored error bars); the best model simulation is seen as a colored solid line; the model uncertainty as a colored semi-transparent overlay. The $x$-axis represents time in seconds, and the $y$-axis is the normalized vessel diameter change $(\Delta \%)$. 


\subsubsection{The dynamic behavior observed can be explained by two distinct dilation processes}

The arteriolar responses transition from a one peak (Fig. 3A) to a two-peak response (Fig. 3C), as the stimulation length increases. In other words, for the short stimulation, the response rises to a peak amplitude value around 2-3 seconds, followed by a decline to baseline. For the two longer (10 $\mathrm{s}$ and $30 \mathrm{~s}$ ) stimulations, a quick initial dilation is followed by a small cessation to a plateau value around 10 $\mathrm{s}$ (Fig. 3B-C). At $10 \mathrm{~s}$, the stimulation ends in one case, with a minor dilation before returning to baseline diameter (Fig. 3B). However, for the $30 \mathrm{~s}$ stimulation, a new dilation phase occurs, consistently dilating until the end of the stimulation (Fig. $3 \mathrm{C}, \mathrm{t}=10-30 \mathrm{~s}$ ). Finally, after stimulus cessation for the longest stimulation, but not for the shorter stimulations, a post-peak undershoot in the arteriolar diameter is observed before returning to baseline (Fig. 3C, $t \sim 50 \mathrm{~s}$ ).

As seen in Fig. 3A-C, these complex dynamic behaviors between different stimulation lengths are captured by the model. It is therefore interesting to examine by which mechanisms the model produces these behaviors. In Figure 4, we illustrate how the model simulations can provide such an examination. Model simulations are shown for all three stimulation lengths: $0.125 \mathrm{~ms}$ (Fig. 4A \& E), 10 $\mathrm{s}$ (Fig. 4B \& F) and $30 \mathrm{~s}$ (Fig. 4C \& G). Furthermore, the top three figures correspond to the rapid neuronal responses (Fig. $4 \mathrm{~A}-\mathrm{C}$ ), and the bottom three figures correspond to the impact of the vasoactive substances: NO, PGE2, NPY (Fig. 4E-G). In addition, the simulations are shown for all three neuronal types and corresponding vasoactive substances: pyramidal neurons and $\mathrm{PGE}_{2}$ (green), NO interneuron and NO (blue), and NPY interneurons and NPY (red). Finally, the resulting behavior (Fig. 4E-G, black dashed line) is produced by the summation of the three impacts, where NPY is vasoconstrictive (negative), and $\mathrm{PGE}_{2}$ and $\mathrm{NO}$ are vasodilative (positive). To help navigate these different simulations, we have placed a simplified view of the model in the middle (Fig. 4D).

With these interpretations in place, we can now understand how the model produces the complex dynamic behaviors observed in data. It is easiest to follow the chain of events by starting at the last step before the observed behavior. The response to a short stimulation is produced by the fast release of NO, which produces the initial peak in the arteriolar response (Fig. 4F-G, blue line) and which thereafter simply declines. The other two signaling pathways are slower and therefore negligible. Also for the longer stimulations, the NO behavior is important. For these stimulations, the NO response culminates quickly, where after the impact of NO falls which causes a decrease after the first peak. However, for longer stimulations this decrease is replaced by a new increase, which is caused by the rise of the slower $\mathrm{PGE}_{2}$ signaling arm from the pyramidal neuron (Fig. 4F-G, green line). In other words, the $\mathrm{PGE}_{2}$ arm is responsible for the second dilation phase observed for the $30 \mathrm{~s}$ stimulation (Fig. 4G, green line). Finally, the NPY signaling arm is only important to explain the post-stimulus undershoot observed in the $30 \mathrm{~s}$ stimulus paradigm as it is so slow that it requires such long stimulation to become larger than the two other arms (Fig. 4G, red line is above the blue and green for $t \sim 50 \mathrm{~s}$ ). In summary, the initial peak is explained by the fast $\mathrm{NO}$ arm, the second peak is explained by the slower PGE 2 arm, and the post-peak undershoot is explained by the even slower NPY arm.

These different dynamics in the three arms occur at the downstream secretion level, but must be initiated by corresponding changes at the rapid neuronal level. This rapid level is depicted in Figure $4 \mathrm{~A}-\mathrm{C}$. For the shortest stimulation (Fig. 4A), the NO interneurons are here the fastest, and pyramidal neurons and NPY interneurons are equally fast. For the two longer stimulations (Fig. 4B-C), the electrophysiology reaches a steady-state within 1 second, where-after no changes occur until the stimulation ends. Since the secretion of NPY is much slower than $\mathrm{PGE}_{2}$, and since both these dynamics occur over many seconds, the differences in dynamics at the downstream secretion level is almost exclusive explained by the difference in intracellular signaling. The only difference concerns the NO dynamic, which has a peak and decline at the secretion level, which is caused by a corresponding rise and fall at the electrophysiology level. 


\section{Neuronal response}
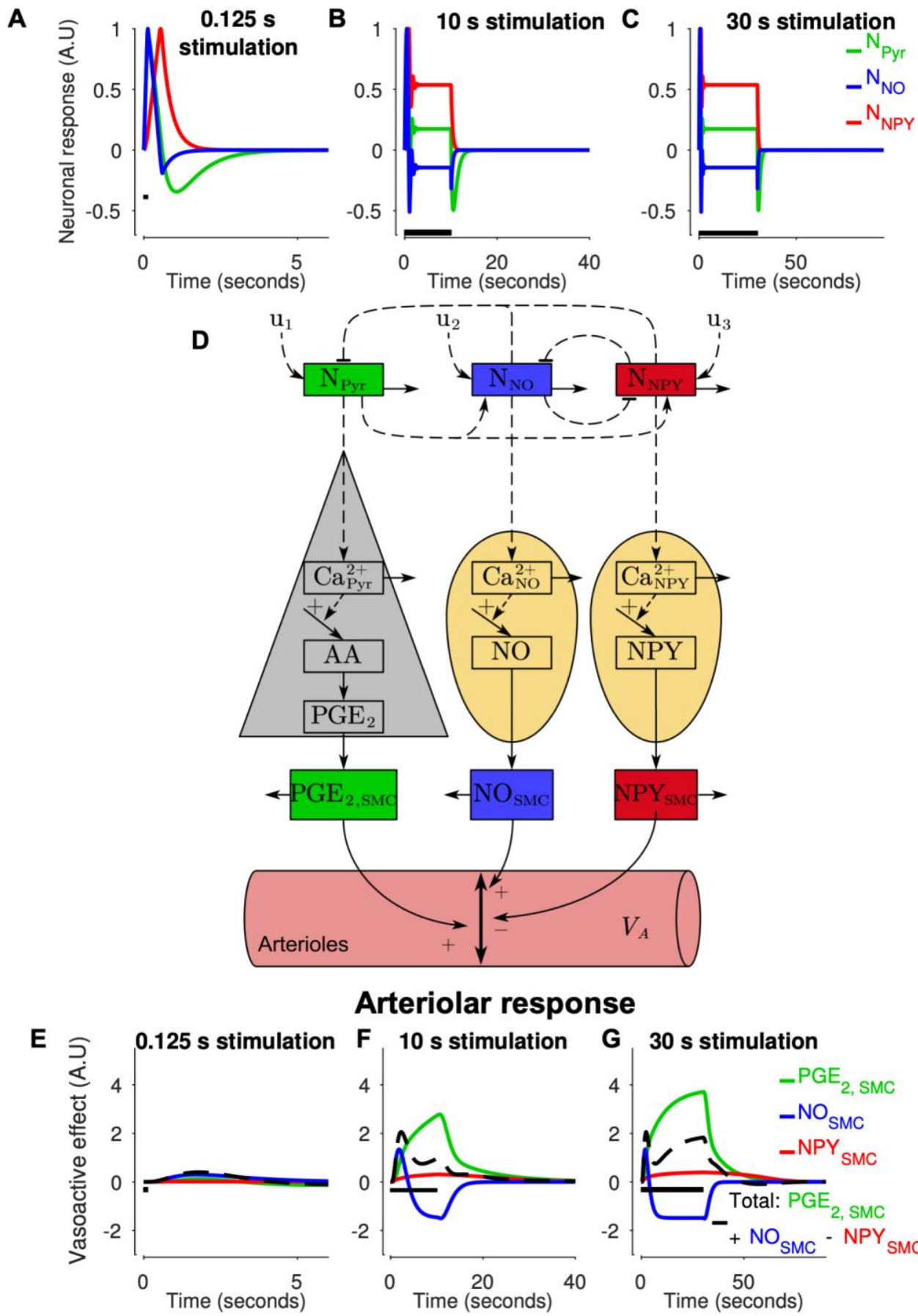

\section{Arteriolar response}
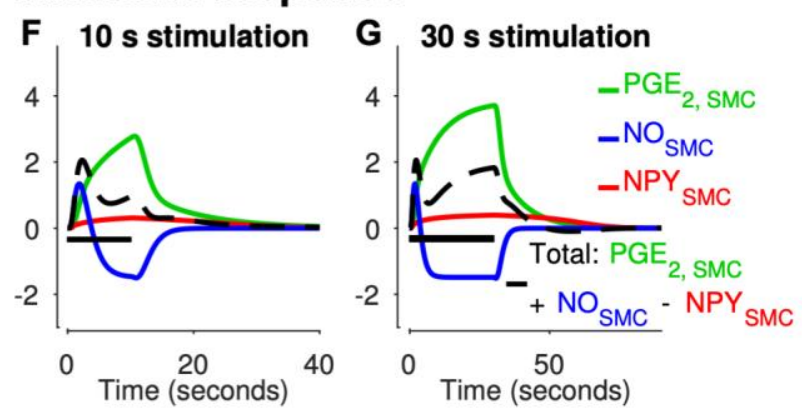

Figure 4 Model explanation (with the relevant model states highlighted in D) of the dynamic arteriolar behavior observed in experimental data from Drew et al. [75]. Model simulations for the three stimulus durations: $125 \mathrm{~ms}(\mathbf{A} \& \mathbf{E}), 10 \mathrm{~s}(\mathbf{B} \& \mathbf{F})$, and $30 \mathrm{~s}(\mathbf{C} \& \mathbf{G})$ are shown. For each stimulus: the dynamic of the neuronal states $\mathrm{N}_{\mathrm{Pyr}}, \mathrm{N}_{\mathrm{NO}}, \mathrm{N}_{\mathrm{NPY}}(\mathbf{A}-\mathbf{C})$, and the arteriolar response $(\mathbf{E}-\mathbf{G})$. For each graph: model simulation (colored lines); stimulus length (black bar). The $\mathrm{x}$-axis represents time in seconds, and the $\mathrm{y}$-axis is the change in neuronal states (A.U) for B-D, and the vasoactive effect on the arteriolar compartment (A.U) for E-G. 
In our previous computational work (see [41]), we developed a model capable of estimating and predicting the experimental data found in Uhlirova et al. [55]. To verify that this extended model still maintains a good agreement and predictive power to that experimental data, we re-did the model training and model predictions in [41]. The Uhlirova study reports arteriolar diameter changes in mice evoked by different stimuli. These different stimuli include for instance $O G$ and sensory stimulation, where OG can stimulate either pyramidal neurons or inhibitory interneurons separately. They do this cell-specific stimulation with and without pharmacological perturbations, using the NPY receptor inhibitor BIBP, and the glutamatergic signaling inhibitors CNQX and AP5. These pharmacological perturbations cut off the cross-talk between NPY and the vasculature and the cross-talk between pyramidal neurons and interneurons, respectively. Furthermore, they also study the animals during awake and anesthesia conditions. In [41], our model can explain all this data with the same parameters, and can also predict independent data not used for training. Our updated model has the same capability (see Supplementary Material S1.2; Fig. S2), and these results e.g., support the notion that NPY acts vasoconstrictively, and causes the post-peak undershoot. 


\subsubsection{Hemoglobin and BOLD measures in awake mice}

Next, we analyzed experimental data from the study of Desjardins et al. [76]. In brief, the study reports hemodynamic measures of blood oxygenation ( $\mathrm{HbO}, \mathrm{HbR}, \mathrm{HbT})$ from three different stimulations: 1$)$ OG stimulation of inhibitory interneurons; 2) OG stimulation of pyramidal neurons, and 3) sensory whisker stimulation using air puffs. Each of these three paradigms had a short (OG: $100 \mathrm{~ms}$ light pulse; sensory: $2 \mathrm{~s}$ of air puffs at $3-5 \mathrm{~Hz}$ ) and long (OG: $20 \mathrm{~s}$ of $100 \mathrm{~ms}$ light pulses at $1 \mathrm{~Hz}$; sensory: $20 \mathrm{~s}$ of air puffs at $3-5 \mathrm{~Hz}$ ) duration. They also reported fMRI-BOLD data for the long OG stimulation of pyramidal neurons. We implemented these stimulus paradigms in the model and assumed a 100 ms air puff delivered at $3 \mathrm{~Hz}$ for the sensory stimulus. To capture the variability in frequency, which gives rise to an observable difference in stimulation strength between the two sensory experiments (Fig. 5C, F), we allowed the input parameters $\left(k_{\mathrm{u}, \mathrm{i}}\right)$ to change between the short and long duration of the sensory stimulation but could keep the same values for both of the OG paradigms (see Discussion 4.2). Furthermore, in the model, we assumed a baseline blood volume concentration of $100 \mu \mathrm{M}$ hemoglobin, in line with previous models $[35,37,85]$.

With these data and model settings, the model was trained to experimental data of hemoglobin dynamics for all three stimulation paradigms simultaneously (Fig. 5A-F, red/blue/green symbols), and the BOLD data was left out to be used for model validation (Fig. 5G, magenta symbols). The model achieved a quantitative acceptable agreement to experimental data (Fig. $5 \mathrm{~A}-\mathrm{F}$, red/blue/green lines) after parameter estimation $\left(J_{l s q}\left(\hat{\theta}_{H 3}\right)=338.3739\right.$, cut-off: $\chi^{2}$ (354 data points) $=397.87$. The degrees of freedom for the confidence interval was $d f_{H 4}=43$, which is equal to the number of estimated parameters (Fig. 5A-F, shaded areas).

To validate the model and avoid overfitting of the parameters, we used the model to generate predictions of a BOLD response for a $20 \mathrm{~s}$ OG stimulation of pyramidal neurons. These predictions are depicted as the magenta shaded area in Fig. 5G. The corresponding experimental data (Fig. 5G, magenta symbols) lie within the model prediction bounds. The validation of the model is supported by a $\chi^{2}$ test, which does not reject the model with respect to validation data $\left(J_{l s q}\left(\hat{\theta}_{H 3}\right)=17.6017\right.$, cut-off: $\chi^{2}(23$ data points $\left.)=35.1725\right)$.

In summary, all results up until now taken together show that our model structure provides an acceptable explanation for a wide variety of aspects observable in mice. Figures 3-4 unravel the role of three regulatory arms: $\mathrm{NO}$ interneurons are responsible for the rapid rise, $\mathrm{PGE}_{2}$ from pyramidal neurons are responsible for the second peak that occurs for long stimulations, and NPY interneurons for the post-peak undershoot. All these results are using vessel diameter as a proxy for the NVC control. In Figure 5, we confirm that the same model can explain the NVC control seen using other measures: total hemoglobin serves as a proxy for blood volume, $\mathrm{HbO} / \mathrm{HbR}$ examines the balance between metabolism and blood flow, and the BOLD signal provides a link to the most common noninvasive measure in primates and humans. 


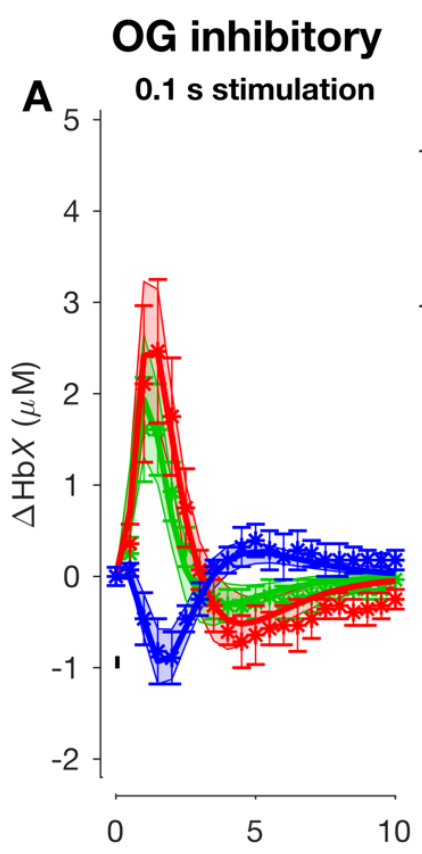

OG excitatory

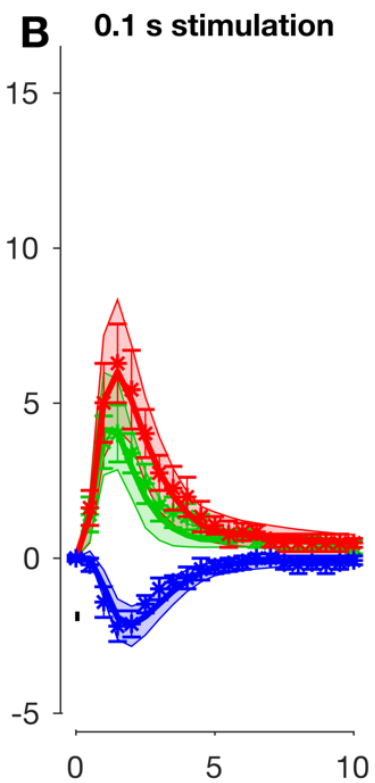

\section{Sensory}
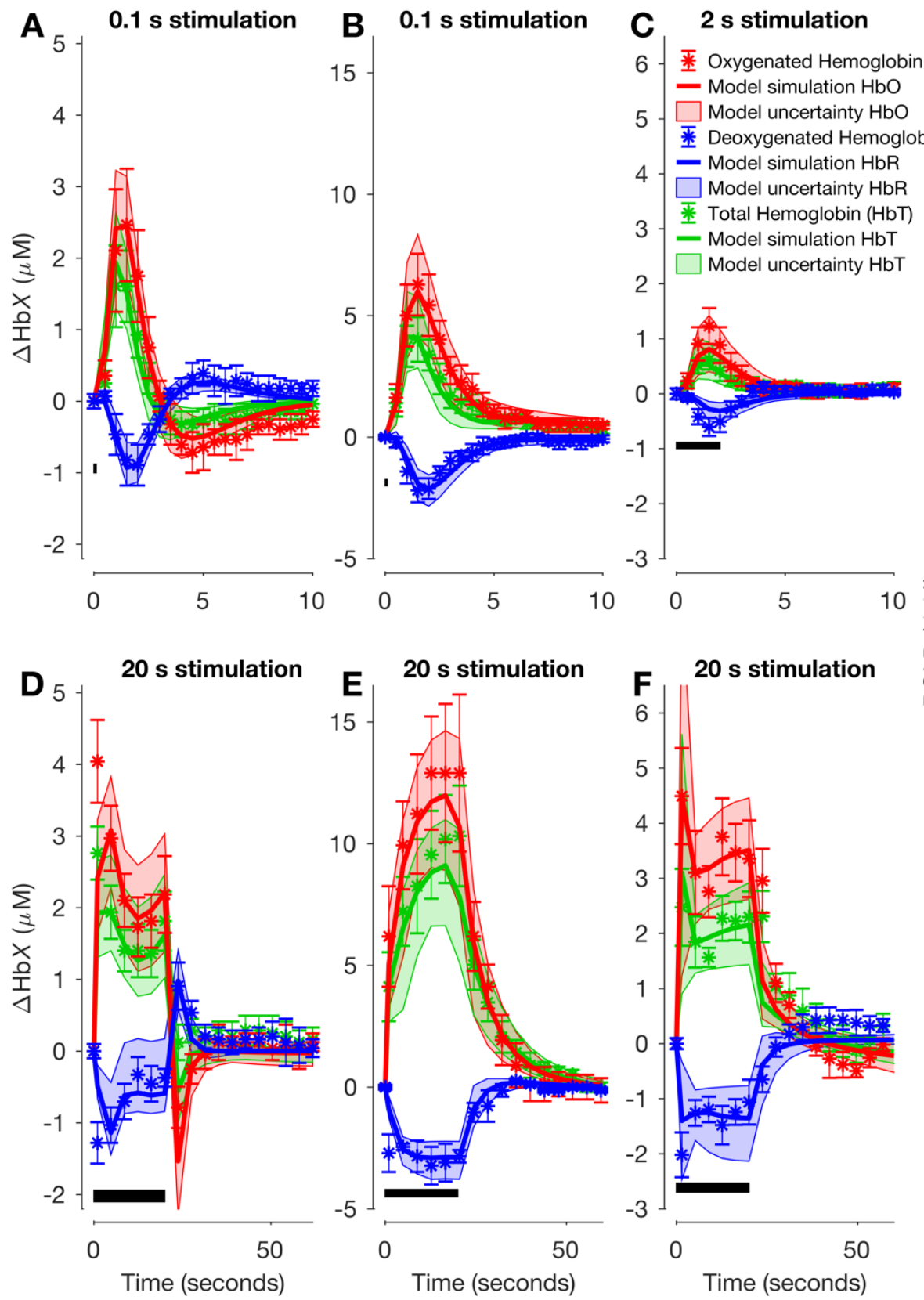

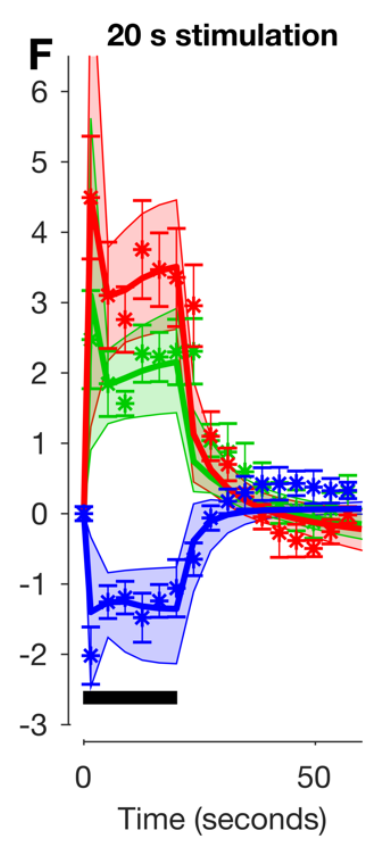

\section{Model Prediction}

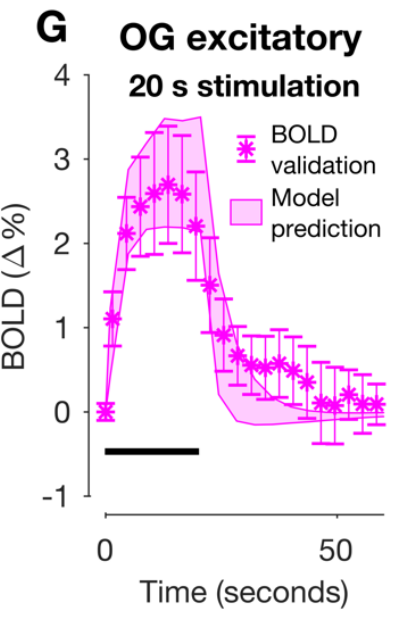

Figure 5 Model estimation to experimental data of hemoglobin changes in mice for three different stimulation types: optogenetic (OG) activation of inhibitory (A \& D) and excitatory (B \& E) neurons, and a sensory stimulation (C \& F). For each stimuli type, a short stimulus (OG: 100 ms of light (A-B); sensory: $2 \mathrm{~s}$ (C)) and long stimulus (OG: $20 \mathrm{~s}$ (D-E); sensory: $20 \mathrm{~s}(\mathbf{F})$ ) was used. This is denoted with the black bar in the bottom left portion of each graph. The shown experimental data are replotted versions of Fig. S5 in the study by Desjardins et al. [76]. For each graph: experimental data consisting of oxygenated hemoglobin ( $\mathrm{HbO}$; red symbols), deoxygenated hemoglobin ( $\mathrm{HbR}$; blue symbols), and total $\mathrm{Hb}$ ( $\mathrm{HbT}$; green symbols); the uncertainty of the experimental data is presented as SEM (colored error bars); the best model simulation is seen as colored solid lines corresponding to respective measurement variable; the model uncertainty as colored semi-transparent overlays; the $\mathrm{x}$-axis represents time in seconds, and the $\mathrm{y}$-axis is the change in hemoglobin concentration $(\mu \mathrm{M})$. G: Model predictions (shaded area) and experimental data (mean \pm SEM, symbols) of a BOLD response to an identical stimulus is shown in E. The validation experimental data were extracted from Desjardins et al. 2019 [76]. 


\subsection{Neuronal and BOLD measures in anesthetized macaques}

\subsubsection{The model agrees with BOLD and LFP data}

We analyzed experimental data from the study of Shmuel et al. 2006 [10], which presents unique data for higher primates, namely macaques. We extracted electrophysiological and BOLD responses induced by a visual task in anesthetized macaques. The task was presented in two ways, inducing both positive and negative responses in the same area (see Section 2.4.3 for details). These data were extracted from Fig. 1D and $2 \mathrm{~A}$ from the original manuscript. The electrophysiological response was related to the local field potential (LFP), which is to a large extent, influenced by pyramidal neuron firing (see review in [86]). Therefore, we assumed the following measurement equation for the model:

$$
L F P=k_{y_{4}} N_{\mathrm{Pyr}}
$$

where $\mathrm{N}_{\mathrm{Pyr}}$ is the phenological activity state of pyramidal neurons, and $k_{y_{4}}$ is an unknown scaling constant. Between a positive and negative response, we allowed the following parameters to be changed: the input stimulus parameters $\left(k_{\mathrm{u}, \mathrm{Pyr}}, k_{\mathrm{u}, \mathrm{NO}}, k_{\mathrm{u}, \mathrm{NPY}}\right)$, where $k_{\mathrm{u}, \mathrm{Pyr}}<0$, to be able to have both positive and negative values; and the parameters governing the inter-neuronal interactions between $\mathrm{N}_{\mathrm{Pyr}}, \mathrm{N}_{\mathrm{NO}}$, and $\mathrm{N}_{\mathrm{NPY}}\left(k_{\mathrm{PF} 1}, k_{\mathrm{PF} 2}, k_{\mathrm{INF} 1}, k_{\mathrm{INF} 2}, k_{\mathrm{IN} 1}, k_{\mathrm{IN} 2}\right)$.

The model was trained to experimental data for both positive (Fig. 6A-B, red symbols) and negative (Fig. 6A-B, blue symbols) responses simultaneously. The model achieved a quantitative acceptable agreement to experimental data (Fig. 6A-B, red and blue solid lines) after parameter estimation $\left(U_{l s q}\left(\hat{\theta}_{H 4}\right)=172.16\right.$, cut-off: $\chi^{2}(160$ data points $\left.)=190.52\right)$. The degrees of freedom for the confidence interval was $d f_{\mathrm{H} 4}=49$, which is equal to the number of estimated parameters (Fig. 6A-B, shaded areas).

As seen, the simulations agree overall well with the experimental data, as almost all experimental data points lie within the model uncertainty area (Fig. 6A-B, compare shaded areas with corresponding symbols). However, for the positive response, the model fails to agree with the first data point of the LFP experimental data during stimulation, not reaching the same amplitude (Fig. $6 \mathrm{~B}, \mathrm{red}, \mathrm{t}=1 \mathrm{~s}$ ). This issue can be mitigated by allowing the parameters governing the neuronal states $\mathrm{N}_{\mathrm{Pyr}}, \mathrm{N}_{\mathrm{NO}}$, and $\mathrm{N}_{\mathrm{NPY}}$, to change between stimulus and post-stimulus periods (as previously done in the computational work of [36], see Discussion 4.2). 

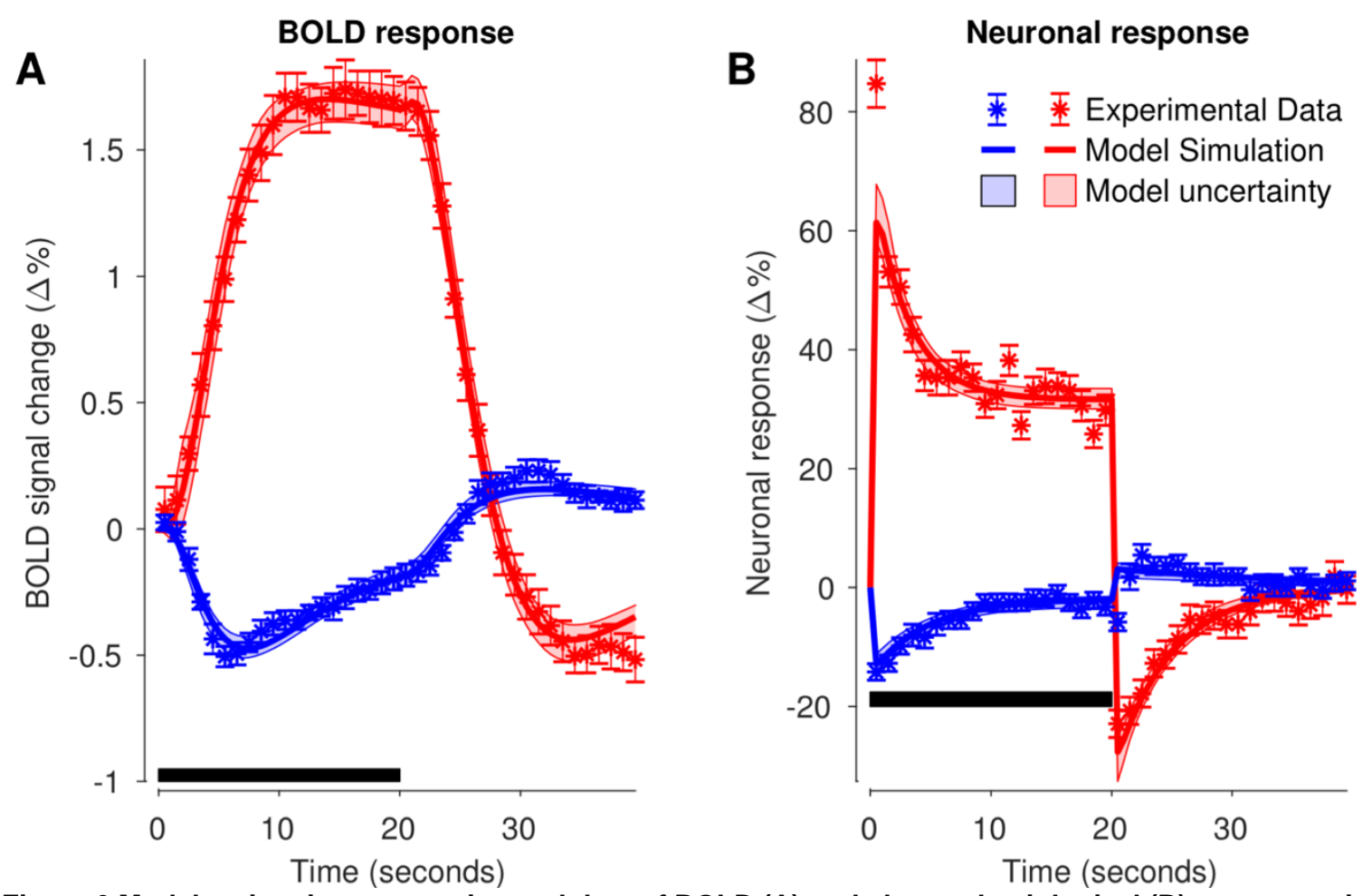

Figure 6 Model estimation to experimental data of BOLD (A) and electrophysiological (B) responses in macaques for two variants of a visual task (blue and red symbols). The task was a $20 \mathrm{~s}$ visual stimulation of $\mathrm{V} 1$ in the visual cortex (marked with the black bar in the bottom left portion of each graph). For each graph: experimental data (colored symbols); The uncertainty of the experimental data is presented as SEM (colored error bars); the best model simulation seen as colored solid lines corresponding to respective measurement observables. The model uncertainty is seen as the semi-transparent colored areas. The $x$-axis represents time in seconds, and the $y$-axis is BOLD signal change $(\Delta \%)$ for $\mathbf{A}$ and LFP expressed as percent change from baseline $(\Delta \%)$ for B. The experimental data were extracted from Fig. 1D \& 2A from Shmuel et al. 2006 [10]. 
bioRxiv preprint doi: https://doi.org/10.1101/2021.03.25.437053; this version posted March $26,2021$. The copyright holder for this preprint (which was not certified by peer review) is the author/funder, who has granted bioRxiv a license to display the preprint in perpetuity. It is made available under aCC-BY-NC 4.0 International license.

\subsection{MR-based hemodynamic measures in awake human}

\subsubsection{The model can describe data used for training and predict independent validation data}

Further, we looked at the study conducted by Huber et al. [77]. We extracted the experimental measurements of CBV, CBF, and BOLD responses in humans, evoked by multiple visual stimuli. The study employed a set of three different flickering checkerboard patterns as the visual stimuli (see Section 2.4.5. for more details). The extracted data originated from figures $4 A-B, 5 B-C$, and $6 C$ in the original paper [77]. The model was trained on experimental data consisting of both positive and negative CBV (Fig. 7A) and BOLD responses (Fig. 7D), as well as experimental data for the total CBV changes for the excitatory (Fig. 7B, black) and inhibitory (Fig. 7C, black) tasks. As in Section 3.2, we allow the input stimulus parameters $\left(k_{\mathrm{u}, \mathrm{i}}\right)$ to change between the three tasks, and the parameters governing the inter-neuronal interactions $\left(k_{\mathrm{PF} 1}, k_{\mathrm{PF} 2}, k_{\mathrm{INF} 1}, k_{\mathrm{INF} 2}, k_{\mathrm{IN} 1}, k_{\mathrm{IN} 2}\right)$ to change between positive and negative responses. Parameter estimation was employed to achieve an acceptable fit to the data $\left(J_{l s q}\left(\hat{\theta}_{H 5}\right)=37.27\right.$, cut-off: $\chi^{2}(122$ data points $\left.)=148.78\right)$. The simulation uncertainty can be seen as the colored shaded areas in Fig. 7A-E. As seen, the model simulations have a good agreement with the experimental data (Fig. 7A and D, shaded areas, and Fig. 7B-C, black shaded areas), as the model uncertainty area overlaps well with the experimental data points. To test the model's predictive qualities, we used the model to generate predictions of CBF for both the positive and negative responses (Fig. 7E), as well as predictions of arterial CBV and venous CBV for both the excitatory (Fig. 7B, red and blue) and inhibitory flickering checkerboard tasks (Fig. 7C, red and blue). There is a good agreement between the model predictions and the experimental data. 

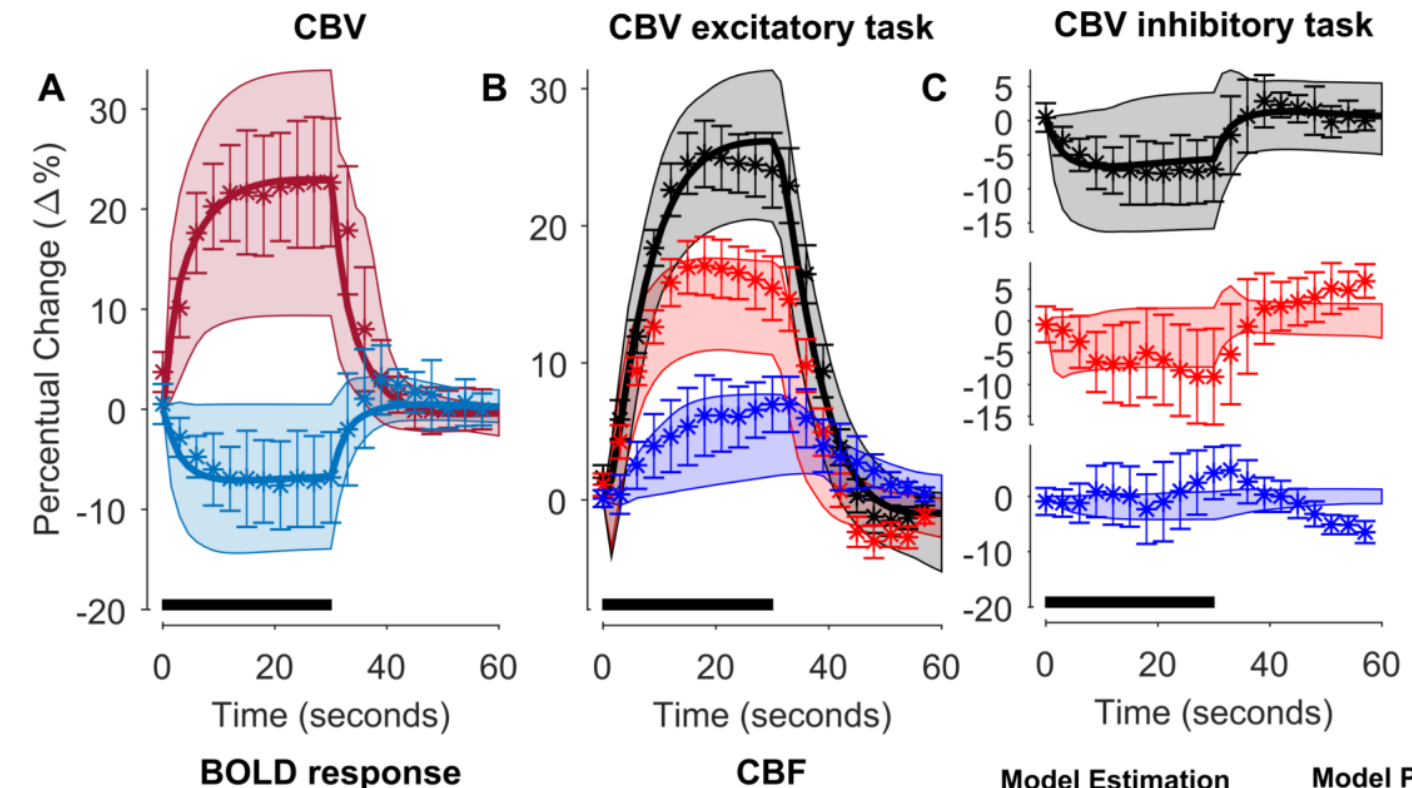

BOLD response
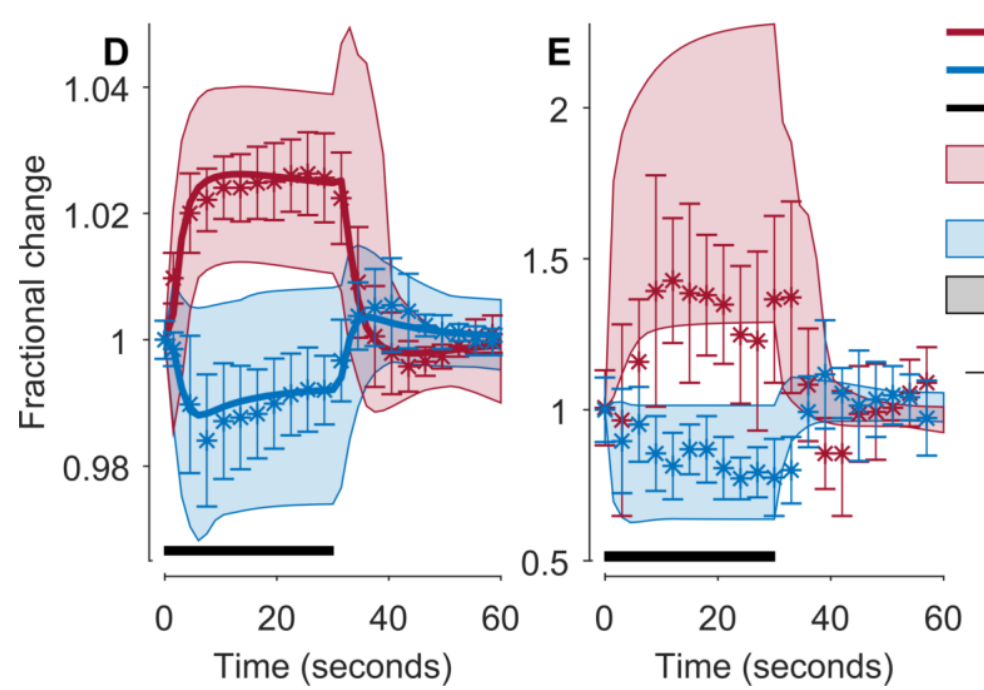
Model Estimation Model Prediction
- Positive BOLD \& CBV uncertainty
- Negative BOLD \& CBV
- Total CBV
Postive BOLD \& CBV
uncertainties Arterial CBV Venous CBV CBF Positive CBF Negative uncertainties
Total CBV uncertainty

Figure 7 Model estimation and prediction to MR-based experimental data in humans for different visual stimulation tasks: The graph contains data for three different tasks: CBV (A), BOLD signal (D), and CBF (E) changes for small flickering checkerboard task, and compartment-specific CBV changes for excitatory (B) and inhibitory (C) flickering tasks. For each graph, the stimulus duration (30 s) is depicted the black bar in the bottom left portion of each graph; experimental data shown with the measurement uncertainty as SEM (colored error bars); the best model simulations is seen as colored solid lines corresponding to respective measurement observables; the model uncertainty is depicted as colored semi-transparent overlays; the $\mathrm{x}$-axis represents time in seconds, and the $y$-axis is the change in fractional $(\mathbf{D}, \mathbf{E})$ or percentual change $(\mathbf{A}-\mathbf{C})$ of the measurement observables. The model prediction uncertainty of CBF $(\mathbf{E})$ and compartment-specific CBV changes $(\mathbf{B}, \mathbf{C})$ is depicted as semi-transparent overlays. The experimental time series are taken from Huber et al. [77]. 


\section{Discussion}

Currently, no existing model of the NVC can simultaneously describe most available NVC data (Fig. 1B, 1D): 1) BOLD, 2) CBV, 3) CBF, 4) Hemoglobin measures, 5) optogenetic and sensory stimulations, 6) pharmacological perturbations including the effect of anesthetics, and 7) phenomenological description of LFP-related signals. Herein, we provide a first such model (Fig. 2). The model can quantitatively explain experimental data used for model training (Figs. 3, 5, 6, 7, S2AE) and correctly predict experimental data not used for training (Fig. 5G; Fig. 7BCE; Fig. S2F-H), from rodents (Figs. 3, 5, S2), primates (Fig. 6), and humans (Fig. 7). This validated model provides a first interactive and inter-connected explanation for how the complicated dynamics and interdependencies in all of these data can be understood. We illustrate this potential by using the model to explain the double peak in arteriolar diameter in response to prolonged stimuli (Fig. 4G, black broken line): the first peak is produced by the rapid NO dilating arm (Fig. 4E-G, blue line), the second peak is produced by the slower $\mathrm{PGE}_{2}$ arm (Fig. 4E-G, green line), and the post-peak undershoot is explained by the even slower NPY arm (Fig. 4F, red line). This new quantitative understanding can also serve as a foundation for a new more integrative approach to analysis of neuroimaging data, which opens the door to many new applications in both basic science and in the clinic.

\subsection{Models of the NVC}

The NVC has previously been mathematically modeled using many different approaches. The earliest models, which still are used in the conventional analysis of BOLD-fMRI data today, are based on the general linear model (GLM), as implemented in software packages like statistical parametric mapping (SPM) $[87,88]$. GLM allows one to identify brain areas with activity, but does so in a way that does not make use of any biological understanding, and thus cannot incorporate any additional data. Such incorporations required more mechanistic models. Simple mechanistic models were presented by Friston et al. [27, 89] and Buxton et al. [28, 29]. These models are commonly referred to as the Balloon model. The Balloon model has become widely used, spawning many subsequent variants and improvements [36, 90-93]. These models typically only describe venous volume changes (as this is the dominant contributor to the BOLD-fMRI signal). One significant shortcoming with these models is that they do not consider the arteriolar volume that is actively regulated in the NVC, and that it primarily is the arteriolar volume that changes upon short event-related stimuli (Fig. 3). To remedy this, some have developed Windkessel-like models, which are electrical circuit equivalent models of pressures, volumes, and flows [34, 35, 94] that can describe all cerebrovascular volumes. In contrast, others have instead opted to exclusively describe arteriolar volume changes, which only are applicable for short stimuli $[95,96]$. Finally, other types of models are based on partial differential equations and detailed diffusion images [97-100]. A common shortcoming for all these mentioned approaches is that these models lack biochemical mechanisms underpinning the intracellular signaling, which translates neural activity to hemodynamic changes. Mathematical models describing these intracellular processes found in the NVC exist [38-40, 101-104], but they are typically connected to the more simplified Balloon model, or lack realistic descriptions of the vascular dynamics. In conclusion, no model that is both based on biochemical mechanisms and which propagates vascular changes using a Windkessel model has previously been described (Fig. 1D). In this work, we present a first such model.

\subsection{Discussion on problematic data points}

While the overall agreement between model and data is statistically acceptable, as the model simulations pass $\chi^{2}$-tests, there are some individual data points that cannot be explained by the model and that warrant some additional comments. In Section 3.1.1, we present the model estimation to experimental data collected from the original research in [75]. While the overall model agreement overlaps well with the experimental data, the model simulation does not agree well with data of venule dilation for the $10 \mathrm{~s}$ stimulus (Fig. 3E, compare symbols with the shaded area). The experimental data exhibits a faster post-stimulus decay, while the venule dilation persists for a longer period of time in the model simulation. The identical behavior is seen in the original manuscript for the vascular model [34] (see Fig. 2F in [34] for the corresponding simulation), which was compared to the same experimental data. The reason why both these models fail to accurately capture the dynamics in Fig. 3E is that the $10 \mathrm{~s}$ stimulus data show a much faster decline after the peak than the $30 \mathrm{~s}$ stimulus data. In the original paper for the vascular model, Barrett and co-workers speculate that this discrepancy in data is due to few measurements on single vessels, and that the 
fast signal decay in $10 \mathrm{~s}$ stimulus data would disappear if averaged over many vessels. Another confounding factor reported along with the experimental data ([75]) is that changes in venule diameter of less than $2 \%$, and capillary diameter changes of less than $7 \%$, could not be captured using their measurement setup. For this reason, the data in Fig. 3D-E are at or below the detection limit, and we therefore view the disagreements with corresponding model simulations to not be a major concern.

In Section 3.1.4, the model simulation fails to reach the first data point in Fig. 5D ( $\mathrm{HbO}, \mathrm{HbR}, \mathrm{HbT})$ and Fig. 5E $(\mathrm{HbR})$. This failure is due to the following inconsistency in the data. Examining the difference between the short and long OG IN stimulation for $\mathrm{HbO}$, we would assume that the first data points up until $t=1 \mathrm{~s}$ would be approximately identical, as only one light pulse should have been emitted in both paradigms. However, for $t=1 \mathrm{~s}$, experimental data points differ by a factor of two. As we assume that the effect of each light pulse is identical between short and long stimulation (since the model does not know the future), the model cannot describe this behavior. For this reason, we choose to keep the model with this shortcoming, since the alternative would be to have different parameters for short and long OG stimulation.

In Section 3.2.1, the model describes all data points except for the first data point in the LFP data for the positive response (Fig. 6B, red). In previous computational work based on the same data [36], parameters were allowed to change between stimulus and post-stimulus periods. This alternative approach gives a satisfactory agreement with data, including the first data point. However, we see no reason for why the parameters should change when the stimulus ends, and we therefore opt to accept the disagreement with the first data point. It is possible that a more non-linear description of LFP would provide a better agreement with this first data point. Again, we choose not to follow such a path, as it might lead to overfitting. A similar disagreement with experimental data can also be observed for the negative LFP response (Fig. 6B, blue). Here, the model simulations instantly return towards baseline upon stimulus cessation, while the experimental data show a $\sim 1 \mathrm{~s}$ delay before rapidly changing. In Havlicek et al. [36], this was compensated for by simply prolonging the stimulation period by $1 \mathrm{~s}$. If our model allowed for a similar prolonging, the same improvement would also be seen in our simulations.

In Section 3.3.1, the model prediction uncertainty for the positive response of CBF has a high uncertainty, and therefore some parts of the prediction uncertainty lies outside of the data (Fig. 7E, red). Further, there might be dynamics in the changes of venous CBV for the inhibitory task, not captured by the model. However, because of the high uncertainty of the data, this discrepancy cannot be concluded (Fig. 7C, blue).

\subsection{Limitations with the current model}

As with all models, there exists a set of limitations and assumptions which are necessary, but still limit the scope of conclusions that can be drawn using the model.

First, our electrical activity description is simplified. More specifically, we use a simple phenomenological relationship between GABAergic interneurons and pyramidal neurons, where pyramidal neurons excite the GABAergic interneurons, which in turn act to inhibit the pyramidal neurons (Eq. 3). An important development would be to integrate this model with common simulators of electrical activity, such as NEURON [105] or NEST [106] (see review in [107]). These models describe how membrane potential fluctuations arise as the result of the opening and closing of ion channels due to glutamatergic and GABAergic signaling. Such an integration would also allow for the description of individual neurons, as there exist structurally and functionally different morphologies of interneurons (see [108] for review), which would be a more realistic description compared to our current lumped approach.

Second, since we describe a wide variety of data, our choice of using the same model structure for all the data has implied some choices. For each experimental data set used in this study, we fitted some of the model parameters in the model specifically to each experimental study, but kept all parameters the same within the same study. More specifically, all parameters are the same for all simulations in Figs. 3-4, 5, 6, 7, S1-S2, but some parameters are different, for instance between Figs. 3 \& 5. Details on this is given in supplementary model files. Some other parameters are not fitted to any of these data but are assumed to be the same across studies. For instance, the vascular volume fractions $V_{i, 0}$ and baseline oxygen transport parameters (see Section 2.2.4) are assumed to be the same across the 
different species. Furthermore, some experimental modalities measure directly on individual arterioles and/or venules (Figs. 3 \& S2), while other modalities measure a lumped estimate over the entire arterial or venous compartment (Figs. 5-7). In the model, we use the same simulation variable to describe both individual and lumped measurements.

Another highly simplified component of our model concerns metabolism. While the metabolism of oxygen occurs in the model, with both a baseline and a stimulus-dependent component, this part could, in principle, be expanded to incorporate the commonly measured metabolites in glia. These metabolites are typically measured using magnetic resonance spectroscopy (MRS), and models capable of describing these interplays already exist $[109,110]$. In addition, there are some known additional mechanisms of the NVC that we have not included. For instance, we do not include the role of astrocytes. Astrocytes have end-feet ideally placed around smooth muscle cells on arterioles and pericytes, and astrocytes are known to regulate the basal tone of arteries [111-114]. The reason we did not include these is that the exact role is unclear, and we did not find useful dynamic data. Another vasoactive pathway involves inward-rectifier $\mathrm{K}^{+}\left(\mathrm{K}_{\mathrm{IR}} 2.1\right)$ channels located on capillary endothelial cells, which open upon extracellular increases of $\mathrm{K}^{+}$as a result of neural activity [115]. The opening of these channels induces hyperpolarization, which propagates rapidly upstream to arteries [115]. We do not include direct actions on the capillaries, as reported in $[50,116]$.

Lastly, in the model, the amount of some states (Eq. 3-7) are specified in arbitrary units, which means that we describe biological values such as concentrations, scaled by unknown scaling constants. Furthermore, the vascular states (Eq. 9-11) are normalized to a baseline value of 1 , in order to improve computational stability and simplify calculations. This particular simplification is inherited from Barrett et al. [34, 42, 43]. Despite these renormalizations, our model can still be used to predict and simulate observations that are possible to capture in experimental data.

\subsection{Strengths and potentials}

The big potential with our model is not only the incorporation of more experimental data than any previous model (Fig. 1D), but that we bypass uncertain parameter values and thus can provide predictions with uncertainty, which can be useful in a variety of contexts. One of the main advantages of these predictions is that they can be used to understand mechanisms, which is illustrated in Figures 4. The simulations in Figure 4 are given by a single parameter vector, producing a single line. This is appropriate because the only question we ask is how the complex bimodal response can be produced by the model. For other types of questions, we want to plot predictions with uncertainty.

One often seeks well-determined predictions, sometimes called core predictions [104, 117], and they are useful for instance when validating the model and designing new experiments [118]. Such validations are done for instance in Fig. 5G or Fig. 7BCE (areas without solid lines). These core predictions can also be used to measure otherwise non-measurable entities, which can be estimated from a combination of available data. One classical example of such model-based "measurements" is the approximate measurement of metabolism, which can be done using the Davis model [119], which uses the combination of BOLD and CBF data. In our much more comprehensive and complete model, many more different data sets can be combined, and more possible features can be estimated. These estimated features can both aid in the understanding of mechanisms in basic research (Fig. 4) and be used to identify new biomarkers which can be used to stratify between different patient groups in the clinic. 


\section{Author contributions}

SS, FE, ME and GC designed and conceived the study. SS carried out the model formulation, data extraction, numerical simulations and analysis, and wrote the draft. HP carried out data extraction and model formulation under supervision of SS. NS performed numerical simulations and analysis under supervision of SS. HP, NS, FE, ME, and GC provided critical revisions and comments on the manuscript.

\section{References}

[1] Raichle ME. The restless brain: how intrinsic activity organizes brain function. Philos Trans $R$ Soc B Biol Sci 2015; 370 : 20140172.

[2] Clarke D, Sokoloff L. Circulation and energy metabolism of the brain. In: Basic Neurochemistry. 6th ed. Philadelphia: Lippincott-Raven, 1994.

[3] Roy CS, Sherrington CS. On the Regulation of the Blood-supply of the Brain. J Physiol 1890; 11: i-xiv, 1-158.

[4] ladecola C. Neurovascular regulation in the normal brain and in Alzheimer's disease. Nat Rev Neurosci 5 2004; 5 : 347-360.

[5] Cauli B, Hamel E. Revisiting the Role of Neurons in Neurovascular Coupling. Front Neuroenergetics 2010; 2: 9.

[6] Attwell D, Buchan AM, Charpak S, et al. Glial and neuronal control of brain blood flow. Nature 2010; 468: $232-243$.

[7] Hillman EMC. Coupling mechanism and significance of the BOLD signal: a status report. Annu Rev Neurosci 2014; 37: $161-181$.

[8] Nippert AR, Biesecker KR, Newman EA. Mechanisms Mediating Functional Hyperemia in the Brain. Neuroscientist 2018; 24: 73-83.

[9] Logothetis NK, Pauls J, Augath M, et al. Neurophysiological investigation of the basis of the fMRI signal. Nature 2001; 412: $150-157$.

[10] Shmuel A, Augath M, Oeltermann A, et al. Negative functional MRI response correlates with decreases in neuronal activity in monkey visual area V1. Nat Neurosci 2006; 9: 569-577.

[11] Ogawa S, Lee TM, Kay AR, et al. Brain magnetic resonance imaging with contrast dependent on blood oxygenation. $P$ Natl Acad Sci Usa 1990; 87: 9868-9872.

[12] ladecola C. The Neurovascular Unit Coming of Age: A Journey through Neurovascular Coupling in Health and Disease. Neuron 2017; 96: 17-42.

[13] Ma H, Zhao M, Harris S, et al. Simultaneous multi-wavelength optical imaging of neuronal and hemodynamic activity. Neuromethods 2014; 88: 237-249.

[14] Kazmi SMS, Richards LM, Dunn AK. Cerebral blood flow imaging with laser speckle contrast imaging. Neuromethods 2014; 88: 287-305.

[15] Shih AY, Drew PJ, Kleinfeld D. Imaging vasodynamics in the Awake mouse brain with two-photon microscopy. Neuromethods 2014; 88: 55-73.

[16] Fenno L, Yizhar O, Deisseroth K. The Development and Application of Optogenetics. Annu Rev Neurosci 2011; 34: 389-412.

[17] Goense JBM, Logothetis NK. Neurophysiology of the BOLD fMRI Signal in Awake Monkeys. Curr Biol 2008; 18: 631640.

[18] Goense J, Merkle H, Logothetis NK. High-resolution $\{\mathrm{fMRI}\}$ reveals laminar differences in neurovascular coupling between positive and negative $\{$ BOLD $\}$ responses. Neuron 2012; 76: 629-639.

[19] Gao Y-R, Ma Y, Zhang Q, et al. Time to wake up: Studying neurovascular coupling and brain-wide circuit function in the un-anesthetized animal. Neuroimage 2017; 153: 382-398.

[20] Franceschini MA, Radhakrishnan H, Thakur K, et al. The effect of different anesthetics on neurovascular coupling. Neuroimage 2010; 51: 1367-1377.

[21] Masamoto K, Kanno I. Anesthesia and the quantitative evaluation of neurovascular coupling. J Cereb Blood Flow Metab 2012; 32: 1233-1247.

[22] Aksenov DP, Li L, Miller MJ, et al. Effects of Anesthesia on BOLD Signal and Neuronal Activity in the Somatosensory Cortex. J Cereb Blood Flow I\& Metab 2015; 35: 1819-1826.

[23] Vanduffel W, Fize D, Mandeville JB, et al. Visual motion processing investigated using contrast agent-enhanced fMRI in awake behaving monkeys. Neuron 2001; 32: 565-77.

[24] Patel GH, Cohen AL, Baker JT, et al. Comparison of stimulus-evoked BOLD responses in human and monkey visual cortex. bioRxiv 2018; 345330 .

[25] Leite FP, Mandeville JB. Characterization of event-related designs using BOLD and IRON fMRI. Neuroimage 2006; 29 : 901-909.

[26] Lindquist MA, Meng Loh J, Atlas LY, et al. Modeling the hemodynamic response function in fMRI: efficiency, bias and mis-modeling. Neuroimage; 45. Epub ahead of print 2009. DOI: 10.1016/j.neuroimage.2008.10.065.

[27] Friston KJ, Mechelli A, Turner R, et al. Nonlinear Responses in \{fMRI:\} The Balloon Model, Volterra Kernels, and Other Hemodynamics. Neuroimage 2000; 12: 466477.

[28] Buxton RB, Wong EC, Frank LR. Dynamics of blood flow and oxygenation changes during brain activation: the balloon model. Magn Reson Med 1998; 39: 855-864.

[29] Buxton RB, Uludalu g K, Dubowitz DJ, et al. Modeling the hemodynamic response to brain activation. Neuroimage 2004; 23: S220-S233.

[30] Stokes GG. ON THE EFFECT OF THE INTERNAL FRICTION OF FLUIDS ON THE MOTION OF PENDULUMS.

[31] Bessonov N, Sequeira A, Simakov S, et al. Methods of Blood Flow Modelling. Math Model Nat Phenom 2016; 11: 125.

[32] Sagawa K, Lie RK, Schaefer J. Translation of Otto frank's paper 'Die Grundform des arteriellen Pulses' zeitschrift für biologie 37: 483-526 (1899). J Mol Cell Cardiol 1990; 22: 253-254.

[33] Westerhof N, Lankhaar JW, Westerhof BE. The arterial windkessel. Medical and Biological Engineering and Computing 2009; 47: 131-141. 
[34] Barrett MJP, Tawhai MH, Suresh V. Arteries dominate volume changes during brief functional hyperemia: Evidence from mathematical modelling. Neuroimage 2012; 62: 482-492.

[35] Huppert TJ, Allen MS, Benav H, et al. A multicompartment vascular model for inferring baseline and functional changes in cerebral oxygen metabolism and arterial dilation. J Cereb Blood Flow I\& Metab 2007; 27: 1262-1279.

[36] Havlicek M, Ivanov D, Roebroeck A, et al. Determining excitatory and inhibitory neuronal activity from multimodal fMRI data using a generative hemodynamic model. Front Neurosci. Epub ahead of print 2017. DOI:

$10.3389 /$ fnins.2017.00616.

[37] Huppert TJ, Jones PB, Devor A, et al. Sensitivity of neural-hemodynamic coupling to alterations in cerebral blood flow during hypercapnia. J Biomed Opt 2009; 14: 44038.

[38] Lundengård K, Cedersund G, Sten S, et al. Mechanistic Mathematical Modeling Tests Hypotheses of the Neurovascular Coupling in fMRI. PLoS Comput Biol 2016; 12: e1004971.

[39] Sten S, Lundengård K, Witt ST, et al. Neural inhibition can explain negative BOLD responses: A mechanistic modelling and fMRI study. Neuroimage 2017; 158: 219-231.

[40] Yucel MA, Devor A, Akin A, et al. The Possible Role of CO(2) in Producing A Post-Stimulus CBF and BOLD Undershoot. Front Neuroenergetics 2009; $1: 7$.

[41] Sten S, Elinder F, Cedersund G, et al. A quantitative analysis of cell-specific contributions and the role of anesthetics to the neurovascular coupling. Neuroimage; 215. Epub ahead of print 2020. DOI: 10.1016/j.neuroimage.2020.116827.

[42] Barrett MJP, Suresh V. Extra Permeability is Required to Model Dynamic Oxygen Measurements: Evidence for Functional Recruitment? J Cereb Blood Flow I\& Metab 2013; 33: 1402-1411.

[43] Barrett MJP, Suresh V. Improving estimates of the cerebral metabolic rate of oxygen from optical imaging data. Neuroimage 2015; 106: 101-110.

[44] Griffeth VEM, Buxton RB. A theoretical framework for estimating cerebral oxygen metabolism changes using the calibrated-BOLD method: Modeling the effects of blood volume distribution, hematocrit, oxygen extraction fraction, and tissue signal properties on the BOLD signal. Neuroimage 2011; 58: 198-212.

[45] Lacroix A, Toussay X, Anenberg E, et al. COX-2-Derived Prostaglandin E2 Produced by Pyramidal Neurons Contributes to Neurovascular Coupling in the Rodent Cerebral Cortex. J Neurosci 2015; 35: 11791-11810.

[46] Cockcroft S. Signalling roles of mammalian phospholipase D1 and D2. Cellular and Molecular Life Sciences 2001; 58: 1674-1687.

[47] Lecrux C, Toussay X, Kocharyan A, et al. Pyramidal neurons are 'neurogenic hubs' in the neurovascular coupling response to whisker stimulation. J Neurosci 2011; 31: 9836-9847.

[48] Davis RJ, Murdoch CE, Ali M, et al. EP4 prostanoid receptor-mediated vasodilatation of human middle cerebral arteries. Br J Pharmacol 2004; 141: 580-585.

[49] Cauli B, Tong X-K, Rancillac A, et al. Cortical GABA interneurons in neurovascular coupling: relays for subcortical vasoactive pathways. J Neurosci 2004; 24: 8940-8949.

[50] Mishra A, Reynolds JP, Chen Y, et al. Astrocytes mediate neurovascular signaling to capillary pericytes but not to arterioles. Nat Neurosci 2016; 19: 1619-1627.

[51] Rancillac A, Rossier J, Guille M, et al. Glutamatergic Control of Microvascular Tone by Distinct GABA Neurons in the Cerebellum. J Neurosci 2006; 26: 6997-7006.

[52] Taniguchi H. Genetic dissection of GABAergic neural circuits in mouse neocortex. Front Cell Neurosci $2014 ; 8: 8$.

[53] Vaughn MW, Kuo L, Liao JC. Effective diffusion distance of nitric oxide in the microcirculation. Am J Physiol Circ Physiol 1998; 274: H1705-H1714.

[54] Kocharyan A, Fernandes P, Tong X-K, et al. Specific subtypes of cortical GABA interneurons contribute to the neurovascular coupling response to basal forebrain stimulation. J Cereb Blood Flow Metab 2008; 28: 221-231.

[55] Uhlirova H, Kilic K, Tian P, et al. Cell type specificity of neurovascular coupling in cerebral cortex. Elife; 5. Epub ahead of print 2016. DOI: 10.7554/eLife.14315.

[56] Bao L, Kopp J, Zhang X, et al. Localization of neuropeptide Y Y1 receptors in cerebral blood vessels. Proc Natl Acad Sci U S A 1997; 94: 12661-6.

[57] Abounader R, Elhusseiny A, Cohen Z, et al. Expression of Neuropeptide Y Receptors mRNA and Protein in Human Brain Vessels and Cerebromicrovascular Cells in Culture. J Cereb Blood Flow I\& Metab 1999; 19: 155-163.

[58] Abounader R, Villemure JG, Hamel E. Characterization of neuropeptide Y (NPY) receptors in human cerebral arteries with selective agonists and the new Y1 antagonist BIBP 3226. Br J Pharmacol 1995; 116: 2245-50.

[59] Tan CMJ, Green P, Tapoulal N, et al. The Role of Neuropeptide Y in Cardiovascular Health and Disease. Front Physiol 2018; 9: 1281.

[60] Michaelis L, Menten ML. Die Kinetik der Invertinwirkung. Biochem Z 1913; 49: 333-369.

[61] Johnson KA, Goody RS. The Original Michaelis Constant: Translation of the 1913 Michaelis-Menten Paper. Biochemistry 2011; 50: 8264-8269.

[62] Calvetti D, Prezioso J, Somersalo E. Estimating hemodynamic stimulus and blood vessel compliance from cerebral blood flow data. J Theor Biol 2019; 460: 243-261.

[63] Vovenko E. Distribution of oxygen tension on the surface of arterioles, capillaries and venules of brain cortex and in tissue in normoxia: An experimental study on rats. Pflugers Arch Eur J Physiol 1999; 437: 617-623.

[64] Cartheuser CF. Standard and pH-affected hemoglobin-O2 binding curves of Sprague-Dawley rats under normal and shifted P50 conditions. Comp Biochem Physiol Comp Physiol 1993; 106: 775-82.

[65] Dash RK, Bassingthwaighte JB. Blood $\mathrm{HbO} 2$ and $\mathrm{HbCO} 2$ dissociation curves at varied O2, CO2, pH, 2,3-DPG and temperature levels. Ann Biomed Eng 2004; 32: 1676-1693.

[66] Roland PE, Eriksson L, Stone-Elander S, et al. Does mental activity change the oxidative metabolism of the brain? $J$ Neurosci 1987; 7: 2373-2389.

[67] Perthen JE, Lansing AE, Liau J, et al. Caffeine-induced uncoupling of cerebral blood flow and oxygen metabolism: A calibrated BOLD fMRI study. Neuroimage 2008; 40: 237-247.

[68] Zhao JM, Clingman CS, Närväinen MJ, et al. Oxygenation and hematocrit dependence of transverse relaxation rates of blood at 3T. Magn Reson Med 2007; 58: 592-597.

[69] Gustard S, Williams EJ, Hall LD, et al. Influence of baseline hematocrit on between-subject BOLD signal change using gradient echo and asymmetric spin echo EPI. Magn Reson Imaging 2003; 21: 599-607.

[70] Sakai F, Nakazawa K, Tazaki Y, et al. Regional cerebral blood volume and hematocrit measured in normal human volunteers by single-photon emission computed tomography. J Cereb Blood Flow Metab 1985; 5: 207-213.

[71] Spees WM, Yablonskiy DA, Oswood MC, et al. Water proton MR properties of human blood at 1.5 Tesla: Magnetic susceptibility, T1, T2* $2^{*}$, and non-Lorentzian signal behavior. Magn Reson Med 2001; 45: 533-542. 
[72] Kreutz C, Raue A, Kaschek D, et al. Profile likelihood in systems biology. FEBS J 2013; 280: 2564-2571.

[73] Maiwald T, Hass H, Steiert B, et al. Driving the Model to Its Limit: Profile Likelihood Based Model Reduction. PLoS One 2016; 11: e0162366.

[74] Rohatgi A. WebPlotDigitizer.

[75] Drew PJ, Shih AY, Kleinfeld D. Fluctuating and sensory-induced vasodynamics in rodent cortex extend arteriole capacity. Proc Natl Acad Sci U S A 2011; 108: 8473-8478.

[76] Desjardins M, Kılıç K, Thunemann M, et al. Awake Mouse Imaging: From Two-Photon Microscopy to Blood Oxygen Level-Dependent Functional Magnetic Resonance Imaging. Biol Psychiatry Cogn Neurosci Neuroimaging 2019; 4: 533-542.

[77] Huber L, Goense J, Kennerley AJ, et al. Investigation of the neurovascular coupling in positive and negative \{BOLD\} responses in human brain at 7 T. Neuroimage 2014; 97: 349-362.

[78] Huber L, Ivanov D, Krieger SN, et al. Slab-selective, BOLD-corrected VASO at 7 tesla provides measures of cerebral blood volume reactivity with high signal-to-noise ratio. Magn Reson Med 2014; 72: 137-148.

[79] Frohlich F, Kaltenbacher B, Theis FJ, et al. Scalable Parameter Estimation for Genome-Scale Biochemical Reaction Networks. PLoS Comput Biol 2017; 13: e1005331.

[80] Frohlich F, Theis FJ, Radler JO, et al. Parameter estimation for dynamical systems with discrete events and logical operations. Bioinformatics 2017; 33: 1049-1056.

[81] Egea JA, Henriques D, Cokelaer T, et al. MEIGO: an open-source software suite based on metaheuristics for global optimization in systems biology and bioinformatics. BMC Bioinformatics 2014; 15: 136.

[82] Maza M, Yuret D. Dynamic hill climbing. Al Expert; 9.

[83] Stapor P, Weindl D, Ballnus B, et al. PESTO: Parameter EStimation TOolbox. Bioinformatics 2018; 34: 705-707.

[84] Ballnus B, Schaper S, Theis FJ, et al. Bayesian parameter estimation for biochemical reaction networks using regionbased adaptive parallel tempering. Bioinformatics 2018; 34: i494-i501.

[85] Huppert TJ, Allen MS, Diamond SG, et al. Estimating cerebral oxygen metabolism from fMRI with a dynamic multicompartment Windkessel model. Hum Brain Mapp 2009; 30: 1548-1567.

[86] Buzsáki G, Anastassiou CA, Koch C. The origin of extracellular fields and currents-EEG, ECoG, LFP and spikes. Nature Reviews Neuroscience 2012; 13: 407-420.

[87] Friston KJ, Holmes AP, Worsley KJ, et al. Statistical parametric maps in functional imaging: A general linear approach. Hum Brain Mapp 1994; 2: 189-210.

[88] Friston KJ. Statistical parametric mapping : the analysis of functional brain images. Amsterdam; Boston: Elsevier / Academic Press, 2007.

[89] Friston KJ, Josephs O, Rees G, et al. Nonlinear event-related responses in fMRI. Magn Reson Med 1998; 39 : 41-52.

[90] Havlicek M, Roebroeck A, Friston K, et al. Physiologically informed dynamic causal modeling of \{fMRI\} data. Neuroimage 2015; 122: 355-372.

[91] Stephan KE, Kasper L, Harrison LM, et al. Nonlinear dynamic causal models for fMRI. Neuroimage 2008; 42: 649-662.

[92] Marreiros AC, Kiebel SJ, Friston KJ. Dynamic causal modelling for fMRI: a two-state model. Neuroimage 2008; 39: 269-278.

[93] Sotero RC, Trujillo-Barreto NJ. Modelling the role of excitatory and inhibitory neuronal activity in the generation of the BOLD signal. Neuroimage 2007; 35: 149-165.

[94] Zheng Y, Johnston D, Berwick J, et al. A three-compartment model of the hemodynamic response and oxygen delivery to brain. Neuroimage 2005; 28: 925-939.

[95] Kim J, Ress D. Arterial impulse model for the $\{B O L D\}$ response to brief neural activation. Neuroimage 2016; 124: 394408.

[96] Kim J, Khan R, Thompson JK, et al. Model of the transient neurovascular response based on prompt arterial dilation. J Cereb Blood Flow Metab Off J Int Soc Cereb Blood Flow Metab 2013; 33: 1429-1439.

[97] Aquino KM, Robinson PA, Drysdale PM. Spatiotemporal hemodynamic response functions derived from physiology. $J$ Theor Biol 2014; 347: 118-136.

[98] Gagnon L, Sakadžić S, Lesage F, et al. Quantifying the Microvascular Origin of BOLD-fMRI from First Principles with Two-Photon Microscopy and an Oxygen-Sensitive Nanoprobe. J Neurosci 2015; 35: 3663-3675.

[99] Sakadžić S, Mandeville ET, Gagnon L, et al. Large arteriolar component of oxygen delivery implies a safe margin of oxygen supply to cerebral tissue. Nat Commun 2014; 5: 5734 .

[100] Gagnon L, Smith AF, Boas DA, et al. Modeling of cerebral oxygen transport based on In vivo microscopic imaging of microvascular network structure, blood flow, and oxygenation. Frontiers in Computational Neuroscience 2016; $10: 82$.

[101] Mathias EJ, Kenny A, Plank MJ, et al. Integrated models of neurovascular coupling and BOLD signals: Responses for varying neural activations. Neuroimage 2018; 174: 69-86.

[102] Dormanns K, van Disseldorp EM, Brown RG, et al. Neurovascular coupling and the influence of luminal agonists via the endothelium. 2015; 364: 49-70.

[103] Mathias EJ, Plank MJ, David T. A model of neurovascular coupling and the BOLD response: PART I. Comput Methods Biomech Biomed Engin 2016; 1-11.

[104] Cedersund G. Prediction Uncertainty Estimation Despite Unidentifiability: An Overview of Recent Developments Uncertainty in Biology: A Computational Modeling Approach. In: Geris L, Gomez-Cabrero D (eds). Cham: Springer International Publishing, pp. 449-466.

[105] Hines M. NEURON - A Program for Simulation of Nerve Equations. In: Neural Systems: Analysis and Modeling. Boston, MA: Springer US, pp. 127-136.

[106] Gewaltig M-O, Diesmann M. NEST (NEural Simulation Tool). Scholarpedia 2007; 2: 1430.

[107] Einevoll GT, Destexhe A, Diesmann M, et al. The Scientific Case for Brain Simulations. Neuron 2019; 102 : 735-744.

[108] Markram H, Toledo-Rodriguez M, Wang Y, et al. Interneurons of the neocortical inhibitory system. Nat Rev Neurosci 2004; 5: 793-807.

[109] Aubert A, Costalat R. A Model of the Coupling between Brain Electrical Activity, Metabolism, and Hemodynamics: Application to the Interpretation of Functional Neuroimaging. Neuroimage 2002; 17: 11621181.

[110] Gruetter R, Seaquist ER, Ugurbil K. A mathematical model of compartmentalized neurotransmitter metabolism in the human brain. Am J Physiol Endocrinol Metab 2001; 281: E100-12.

[111] Rosenegger DG, Tran CHT, Wamsteeker Cusulin Jl, et al. Tonic Local Brain Blood Flow Control by Astrocytes Independent of Phasic Neurovascular Coupling. J Neurosci 2015; 35: 13463.

[112] Filosa JA, Morrison HW, Iddings JA, et al. Beyond neurovascular coupling, role of astrocytes in the regulation of vascular tone. Neuroscience 2016; 323: 96-109. 
bioRxiv preprint doi: https://doi.org/10.1101/2021.03.25.437053; this version posted March 26, 2021. The copyright holder for this preprint (which was not certified by peer review) is the author/funder, who has granted bioRxiv a license to display the preprint in perpetuity. It is made available under aCC-BY-NC 4.0 International license.

[113] Iddings JA, Kim KJ, Zhou Y, et al. Enhanced parenchymal arteriole tone and astrocyte signaling protect neurovascular coupling mediated parenchymal arteriole vasodilation in the spontaneously hypertensive rat. $J$ Cereb Blood Flow Metab 2015; 35: 1127-1136.

[114] Kim KJ, Iddings JA, Stern JE, et al. Astrocyte contributions to flow/pressure-evoked parenchymal arteriole vasoconstriction. J Neurosci 2015; 35: 8245-8257.

[115] Longden TA, Dabertrand F, Koide M, et al. Capillary K+-sensing initiates retrograde hyperpolarization to increase local cerebral blood flow. Nat Neurosci 2017; 20: 717-726.

[116] Hall CN, Reynell C, Gesslein B, et al. Capillary pericytes regulate cerebral blood flow in health and disease. Nature 2014; 508: 55-60.

[117] Cedersund G. Conclusions via unique predictions obtained despite unidentifiability--new definitions and a general method. FEBS J 2012; 279: 3513-3527.

[118] Nyman E, Lindh M, Lovfors W, et al. Mechanisms of a sustained anti-inflammatory drug response in alveolar macrophages unraveled with mathematical modeling. bioRxiv 2020; 2020.04.13.031245.

[119] Davis TL, Kwong KK, Weisskoff RM, et al. Calibrated functional MRI: Mapping the dynamics of oxidative metabolism. Proc Natl Acad Sci U S A 1998; 95: 1834-1839. 
bioRxiv preprint doi: https://doi.org/10.1101/2021.03.25.437053; this version posted March $26,2021$. The copyright holder for this preprint

(which was not certified by peer review) is the author/funder, who has granted bioRxiv a license to display the preprint in perpetuity. It is made available under aCC-BY-NC 4.0 International license.

\section{Supplementary material accompanying}

\section{A multi-data based quantitative model for the neurovascular coupling in the brain.}

Sebastian Sten ${ }^{1,2}$, Henrik Podéus ${ }^{1}$, Nicolas Sundqvist ${ }^{3}$, Fredrik Elinder ${ }^{4}$, Maria Engström ${ }^{1,2 *}$, Gunnar Cedersund $2,3^{*}$

* These authors contributed equally to this work.

1 Department of Health, Medicine and Caring Sciences, Linköping University, Linköping, Sweden

2 Center for Medical Image Science and Visualization (CMIV), Linköping University, Linköping, Sweden

3 Department of Biomedical Engineering, Linköping University, Linköping, Sweden

4 Department of Biomedical and Clinical Sciences, Linköping University, Linköping, Sweden

\section{Correspondence to:}

Gunnar Cedersund

IMT, Linköping University/US

58183 Linköping, Sweden

$+4613282705$

gunnar.cedersund@gmail.com 
The supplementary material contains the following: additional model estimations, and the posterior probability profiles of the parameters for all model estimation.

\section{S1 Additional model estimations}

S1.1 Allowing the stiffness and viscoelasticity parameters between the capillary and venular compartments to change between $10 \mathrm{~s}$ and $30 \mathrm{~s}$ stimulation for data from Drew et al. [1].

\subsection{5 s stimulation $\quad 10 \mathrm{~s}$ stimulation $\quad 30 \mathrm{~s}$ stimulation}

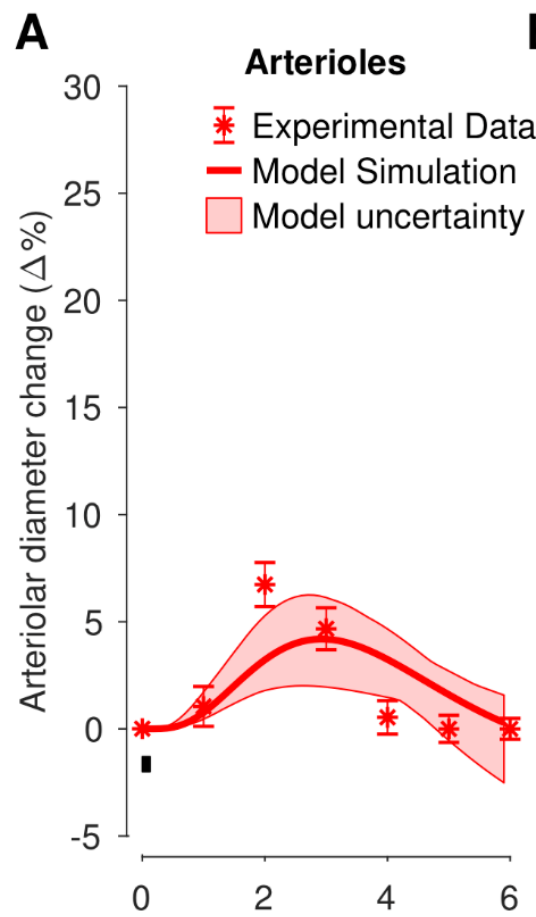

B

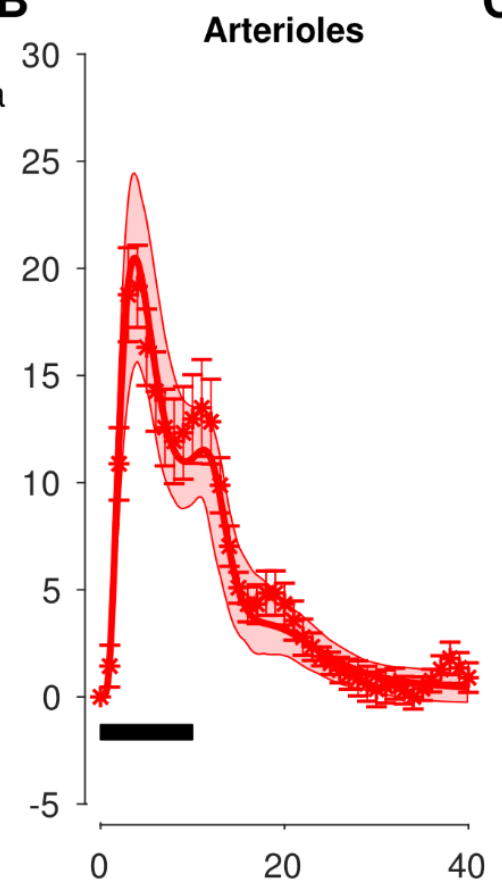

E

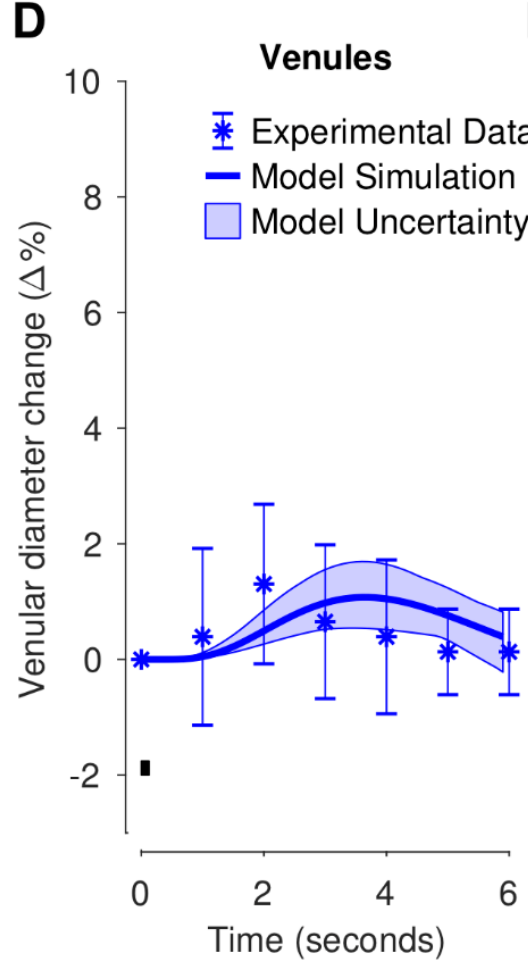

C

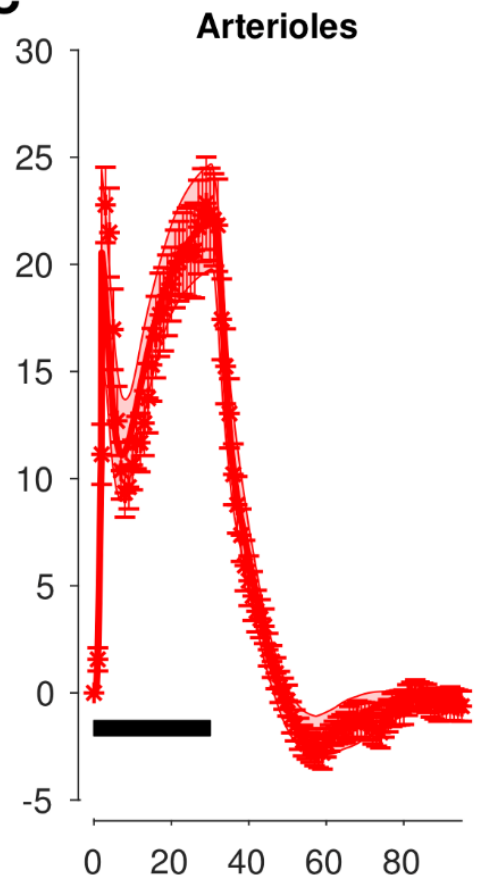

$\mathbf{F}$
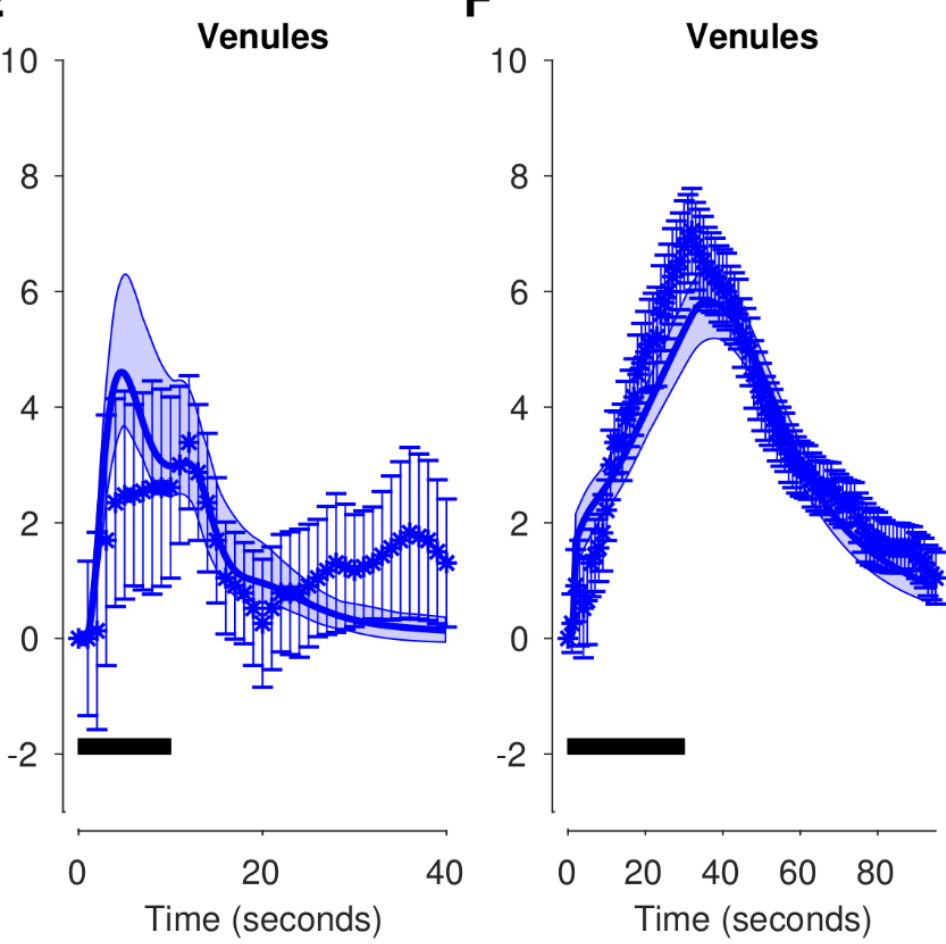

Figure S1 Model estimation to experimental data of arteriolar (A-C) and venular (D-F) volume changes in awake mice for three different sensory stimulation lengths: $125 \mathrm{~ms}$ (A \& D), $10 \mathrm{~s}$ (B \& E), and $30 \mathrm{~s}$ (C \& F). The viscoelasticity and stiffness coefficients of the capillary and venular compartment change at $t=3 s$ for the long stimulation (C \& F). Experimental data are replotted versions of data presented in Fig. $2 \mathrm{C}$ of the original 
manuscript by Drew et al. [1]. The stimulation lengths are denoted with the black bar in the bottom left portion of each graph. For each graph: experimental data (colored symbols); The uncertainty of the experimental data is presented as SEM (colored error bars); the best model simulation is seen as a colored solid line; the model uncertainty as colored semi-transparent overlay. The $x$-axis represents time in seconds, and the $y$-axis is the normalized vessel diameter change $(\Delta \%)$.

\section{S1.2 Model estimations for data from Uhlirova et al.}

The Uhlirova study (see [2]) reports arteriolar diameter changes in mice evoked by different stimuli for awake and anesthesia conditions. To account for the anesthetic effect, we allowed 12 parameters governing the neuronal interactions to change between awake and under anesthesia: the three stimulus-scaling parameters $k_{\mathrm{u}, \mathrm{i}}$; the three decay rate parameters $\sin k_{\mathrm{N}, \mathrm{i}}$ affecting $\mathrm{N}_{\mathrm{i}}$, and finally the six neuronal interaction parameters $k_{\mathrm{PF} 1}, k_{\mathrm{PF} 2}, k_{\mathrm{IN} 1}, k_{\mathrm{IN} 2}, k_{\mathrm{INF} 1}$, and $k_{\mathrm{INF} 2}$. Furthermore, we allow the stimulus scaling parameters $k_{\mathrm{u}, \mathrm{i}}$ to change between $\mathrm{OG}$ and sensory stimulation. See our previous study for more details [3].

The thusly parameterized model was trained on experimental data consisting of all control conditions from the Uhlirova et al. [2] (Fig. S2 left panel A-E, black symbols). The model parameters were fitted to the experimental data, achieving an acceptable agreement with data (Fig. S2 A-E solid thick red lines), evaluated using a $\chi^{2}$-test $\left(J_{l s q}\left(\hat{\theta}_{H S 1}\right)=49.06\right.$, cut-off: $\chi^{2}(190$ data points $)=223.16$ for $\alpha=$ $0.05)$. Next, the uncertainty of the model was approximated using Markov chain Monte Carlo sampling (105 iterations), producing posterior parameter profiles (Fig. S5) and a sample of all $\chi^{2}$ acceptable parameters. A confidence interval (Eq. 33 in the main manuscript) with $\alpha=0.05$ and degrees of freedom $d f_{H 3}=54$ (equal to the number of estimated parameters) was drawn and can be seen as the red shaded areas in Fig. S2A-E.

This confidence interval was used to generate model predictions of pharmacological perturbations. The pharmacological perturbations were: 1) sensory stimulation during application of the NPY receptor Y1 antagonist BIBP (Fig. S2F, red shaded area); 2) OG stimulation of GABAergic interneurons during application of BIBP (Fig. S2G, red shaded area), and, 3) OG stimulation of pyramidal neurons during inhibition of glutamatergic signaling by the administration of the AMPA and NMDA receptor antagonists CNQX and AP5, respectively (Fig. S2H, red shaded area). Correspondingly, experimental data of these perturbations are found in Uhlirova et al. [2], and shown as black symbols in Fig.S2F-H. As seen, the model predictions overlap with experimental data, qualitatively correctly predicting the dynamics observed in the experimental data. 


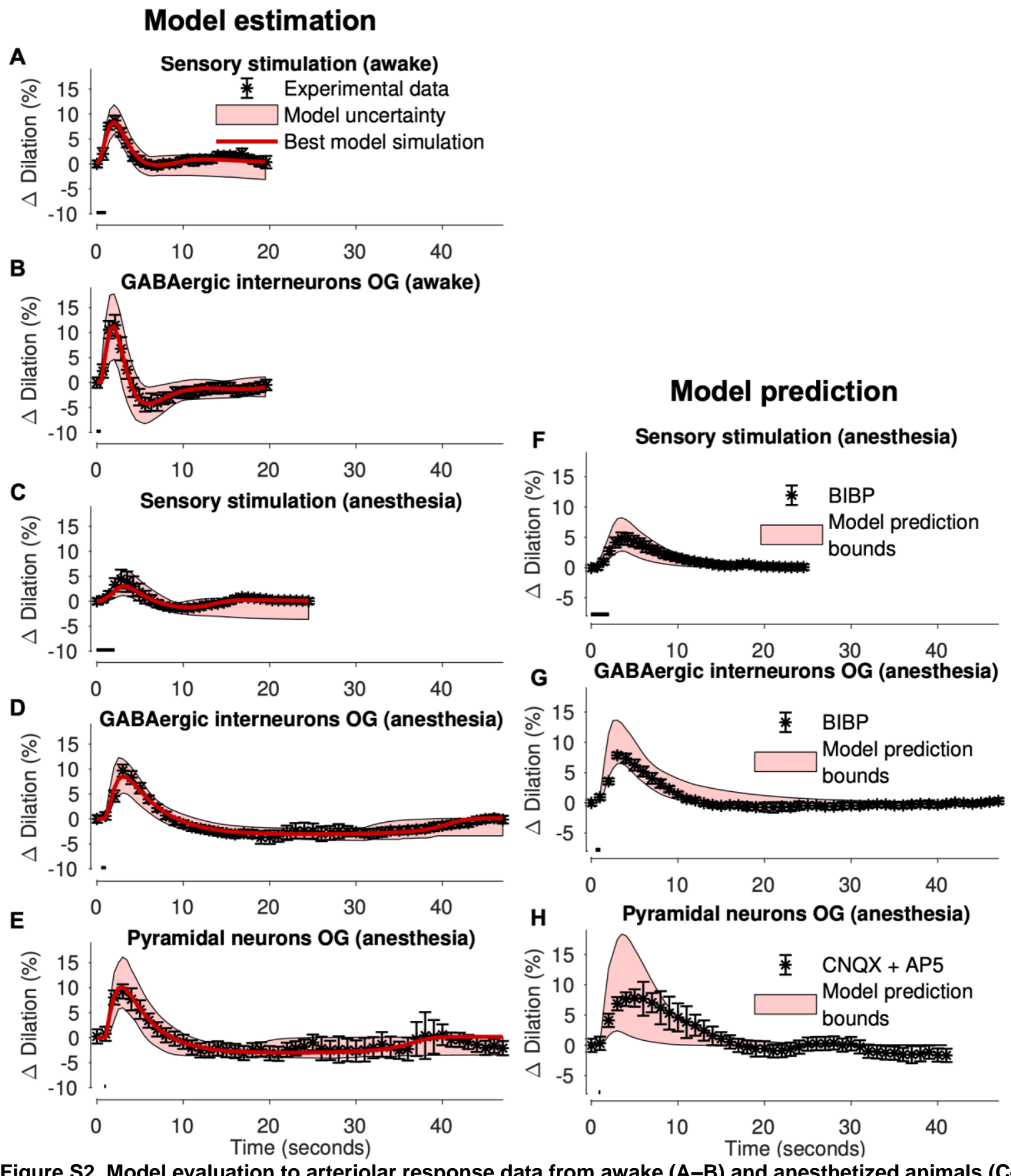

Figure S2. Model evaluation to arteriolar response data from awake (A-B) and anesthetized animals (C-H) for both optogenetic (OG) (B, D-E, G-H) and sensory (A, C, F) stimuli. The model parameters were fitted to data from A-E, while $\mathrm{F}-\mathrm{H}$ was used as validation data. A-E: Model estimation paired with experimental data. For each graph, best estimated model simulation (solid red line) paired with model uncertainty (red shaded areas) compared to experimental data (black symbols, error bars depicting standard error of the mean). The stimulation length is indicated by the black bar in the lower-left portion of each graph. F-H: For each graph, model prediction bounds (red shaded areas) compared to experimental data (black symbols, error bars depicting standard error of the mean). The stimulation length is indicated by the black bar in the lower-left portion of each graph.

Experimental data were extracted from Uhlirova et al. [3]. 


\section{S2 Posterior probability profiles of the model parameters}

\section{S2.1 Posterior probability profiles for model estimation in Figure 3.}
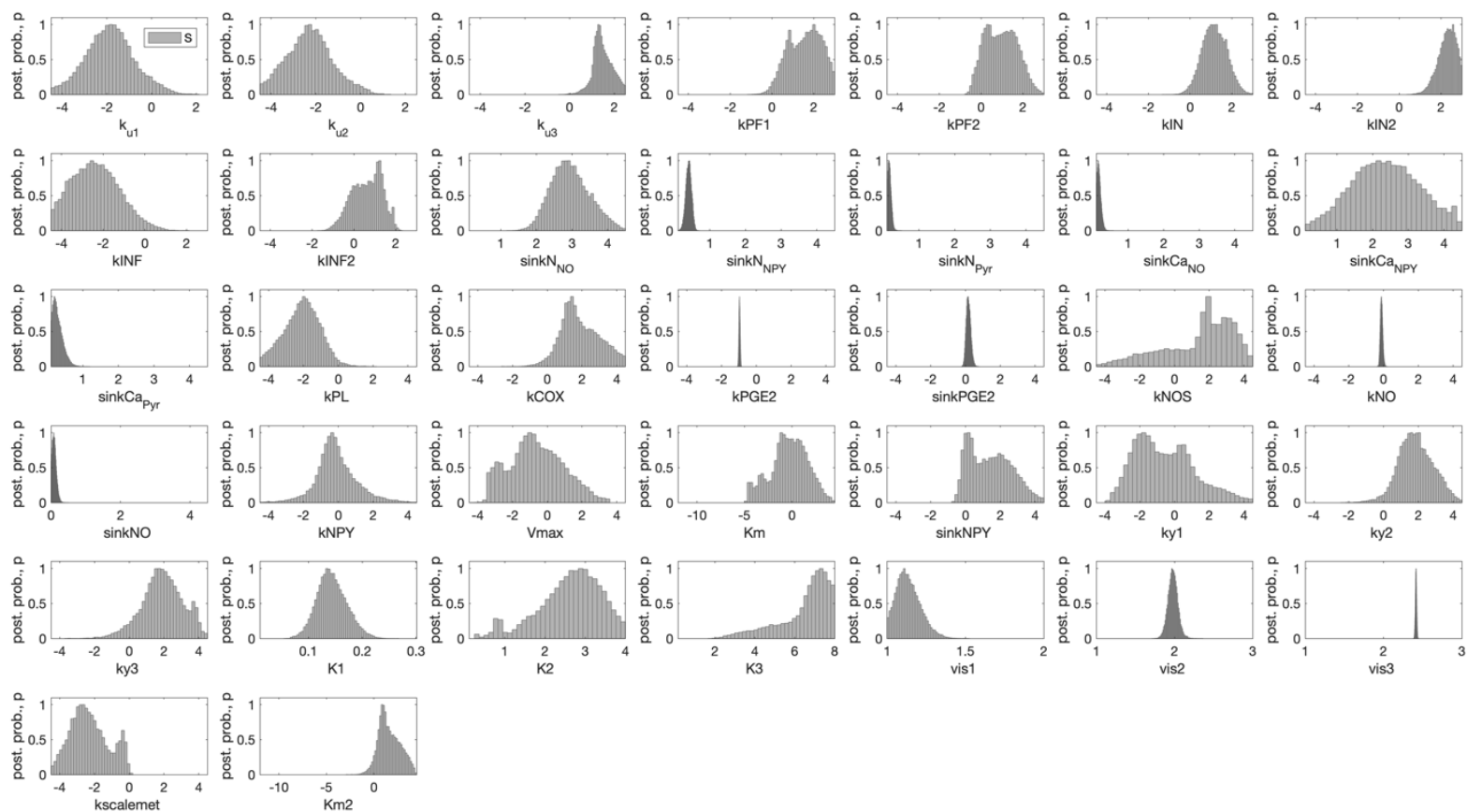

Figure S3. Posterior probability profile ( $y$-axis) for each estimated model parameter ( $x$-axis, $\log _{10}$ space) for the model estimated to data from Drew et al. [1].
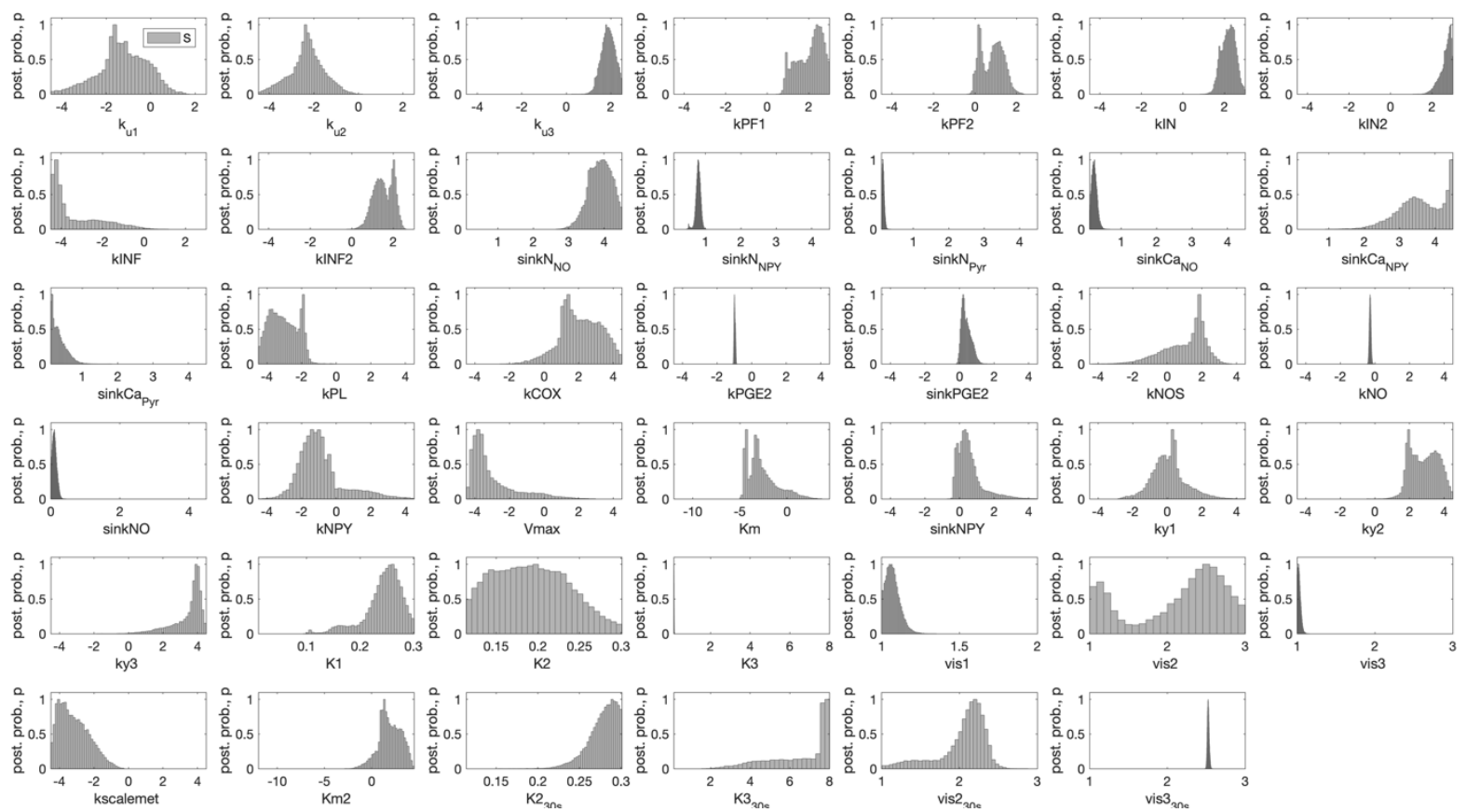

Figure S4. Posterior probability profile ( $y$-axis) for each estimated model parameter ( $x$-axis, $\log _{10}$ space) for the model estimated to data from Drew et al. [1], allowing the viscoelasticity and stiffness coefficients of the capillary and venular compartment change between 10 and $30 \mathrm{~s}$ stimulation. 
S2.2 Posterior probability profiles for model estimation in Figure S2.

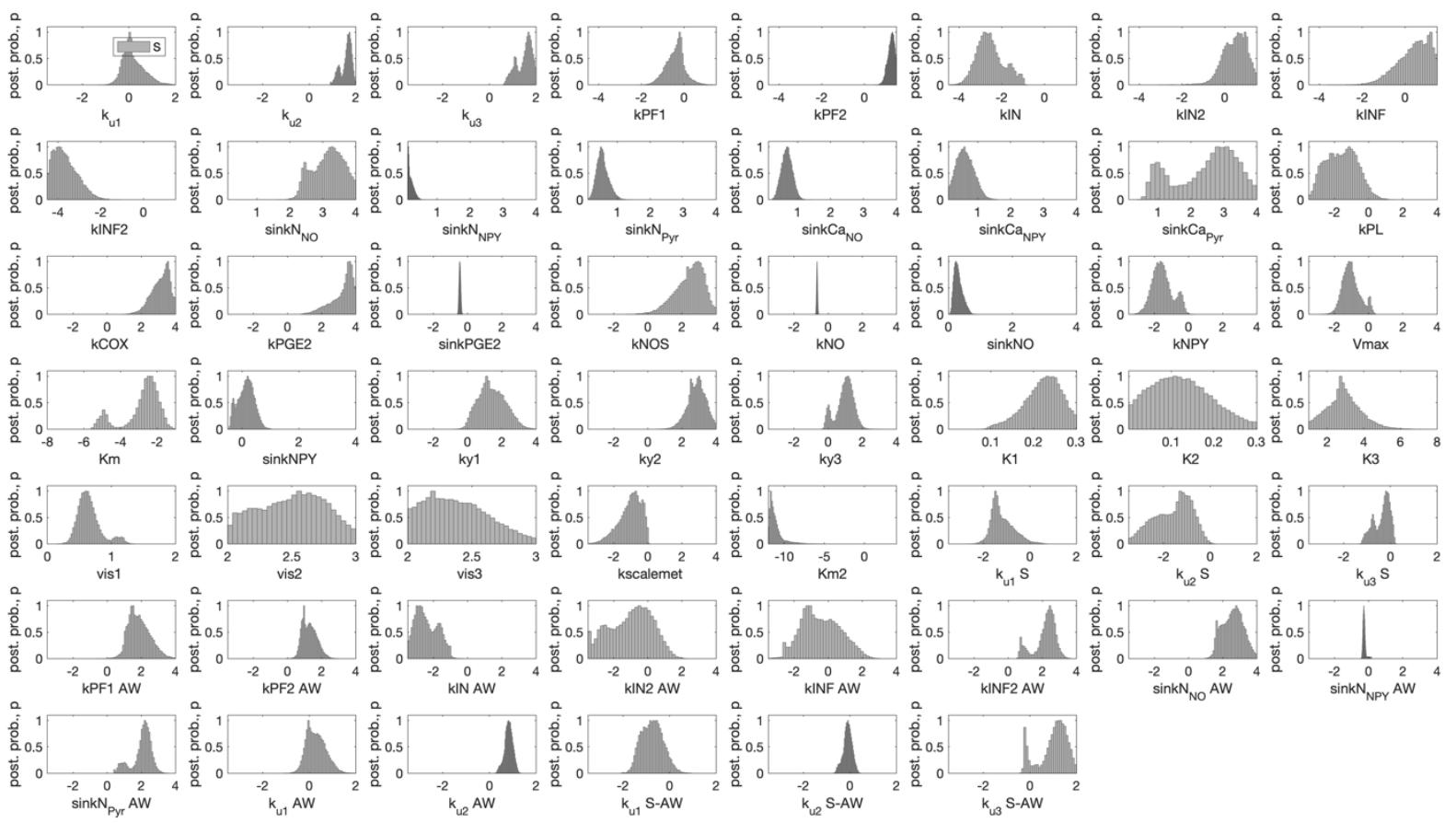

Figure S5. Posterior probability profile ( $y$-axis) for each estimated model parameter ( $x$-axis, $\log _{10}$ space) for the model estimated to data from Uhlirova et al. [2]. Two affixes are used: 'AW' which represent parameter value during awake condition, and ' $S$ ' which represent value for sensory stimulation.

S2.3 Posterior probability profiles for model estimation in Figure 5.

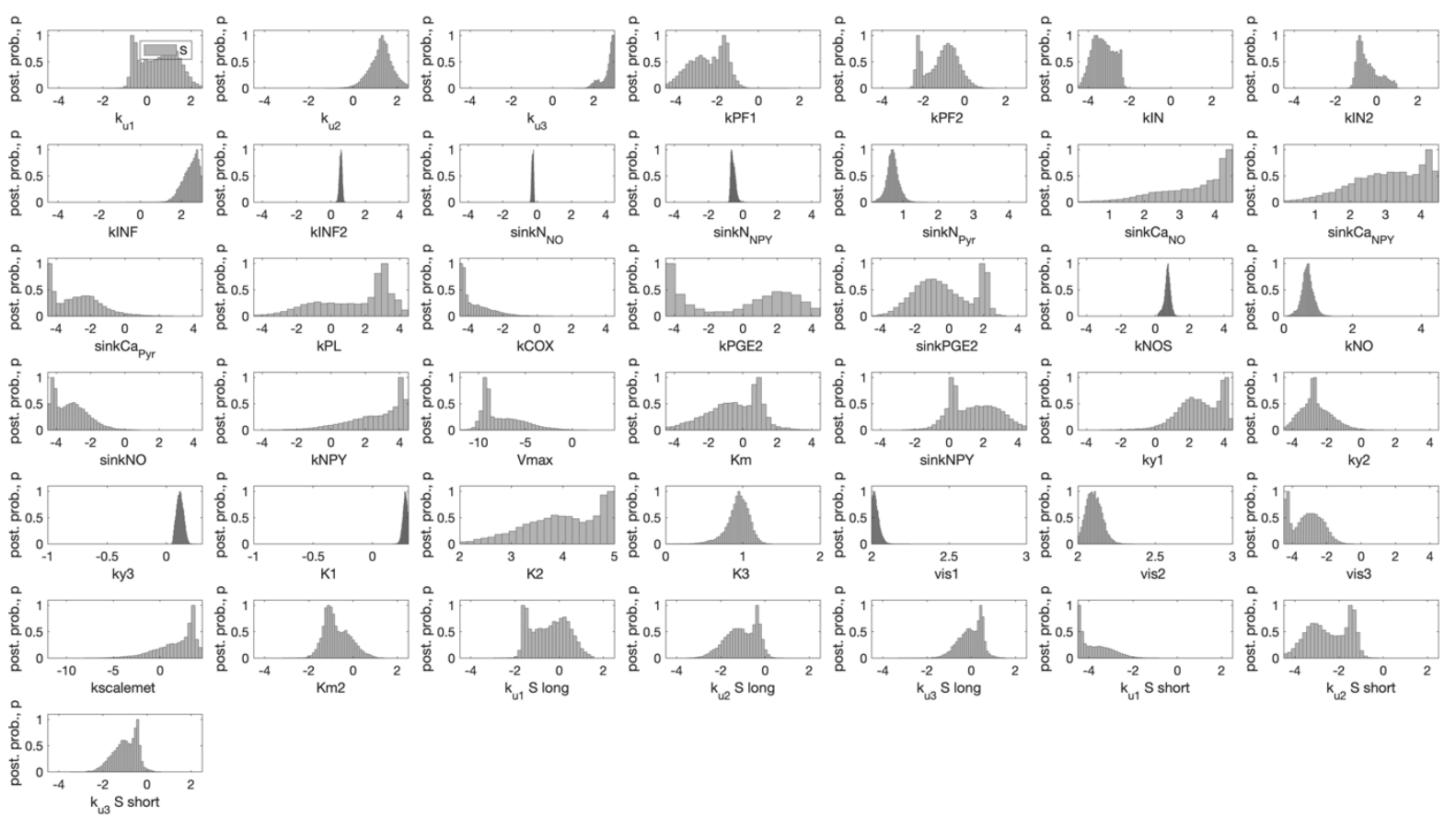

Figure S6. Posterior probability profile ( $y$-axis) for each estimated model parameter ( $x$-axis, $\log _{10}$ space) for the model estimated to data from Desjardins et al. [4]. The affix 'S' represent parameter value for sensory stimulation and is further subdivided into short and long sensory stimulation. 
S2.4 Posterior probability profiles for model estimation in Figure 6.

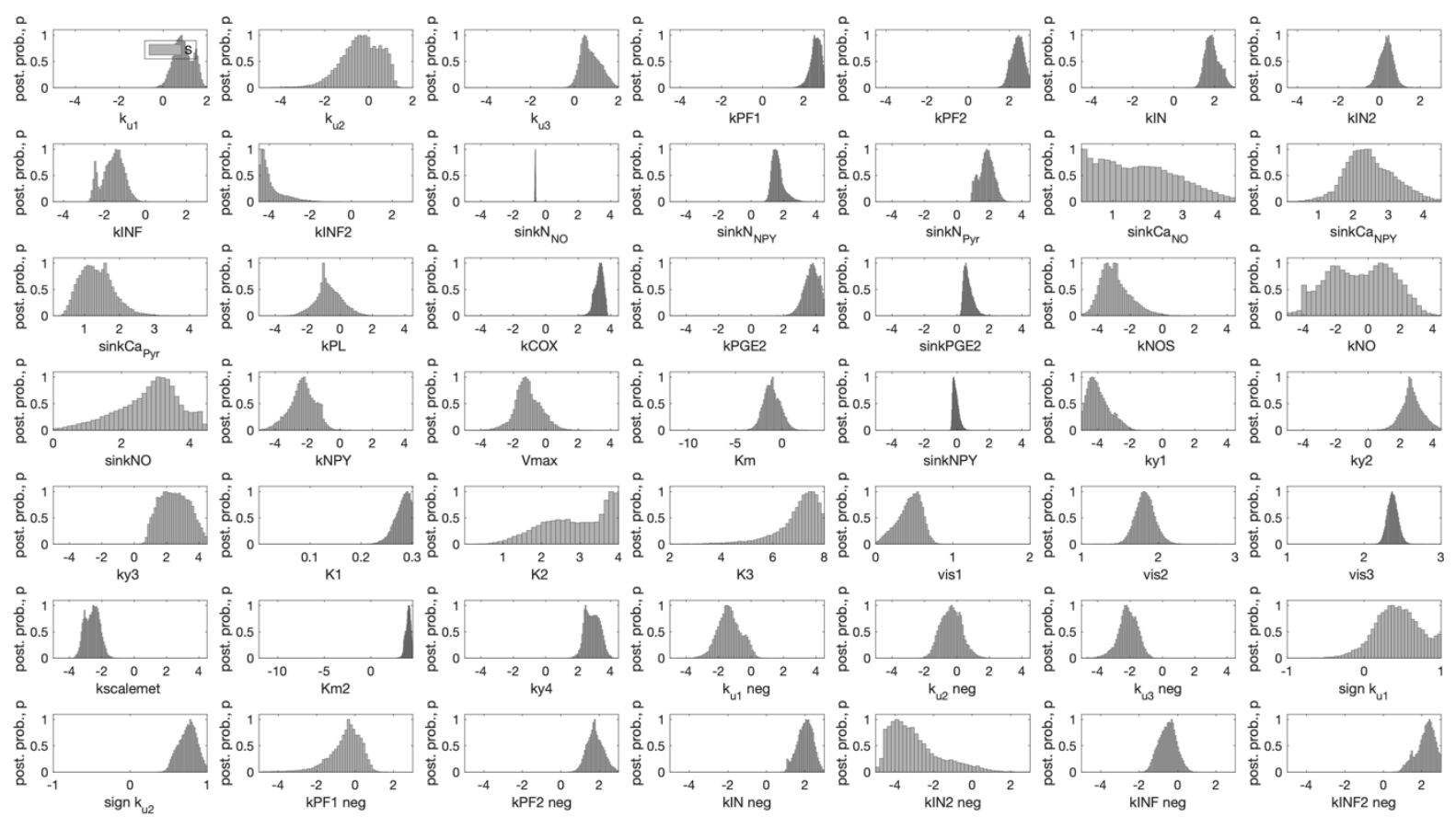

Figure S7. Posterior probability profile ( $y$-axis) for each estimated model parameter ( $x$-axis, $\log _{10}$ space) for the model estimated to data from Shmuel et al. [5]. The affix 'neg' represent parameter value for negative response.

S2.5 Posterior probability profiles for model estimation in Figure 7.

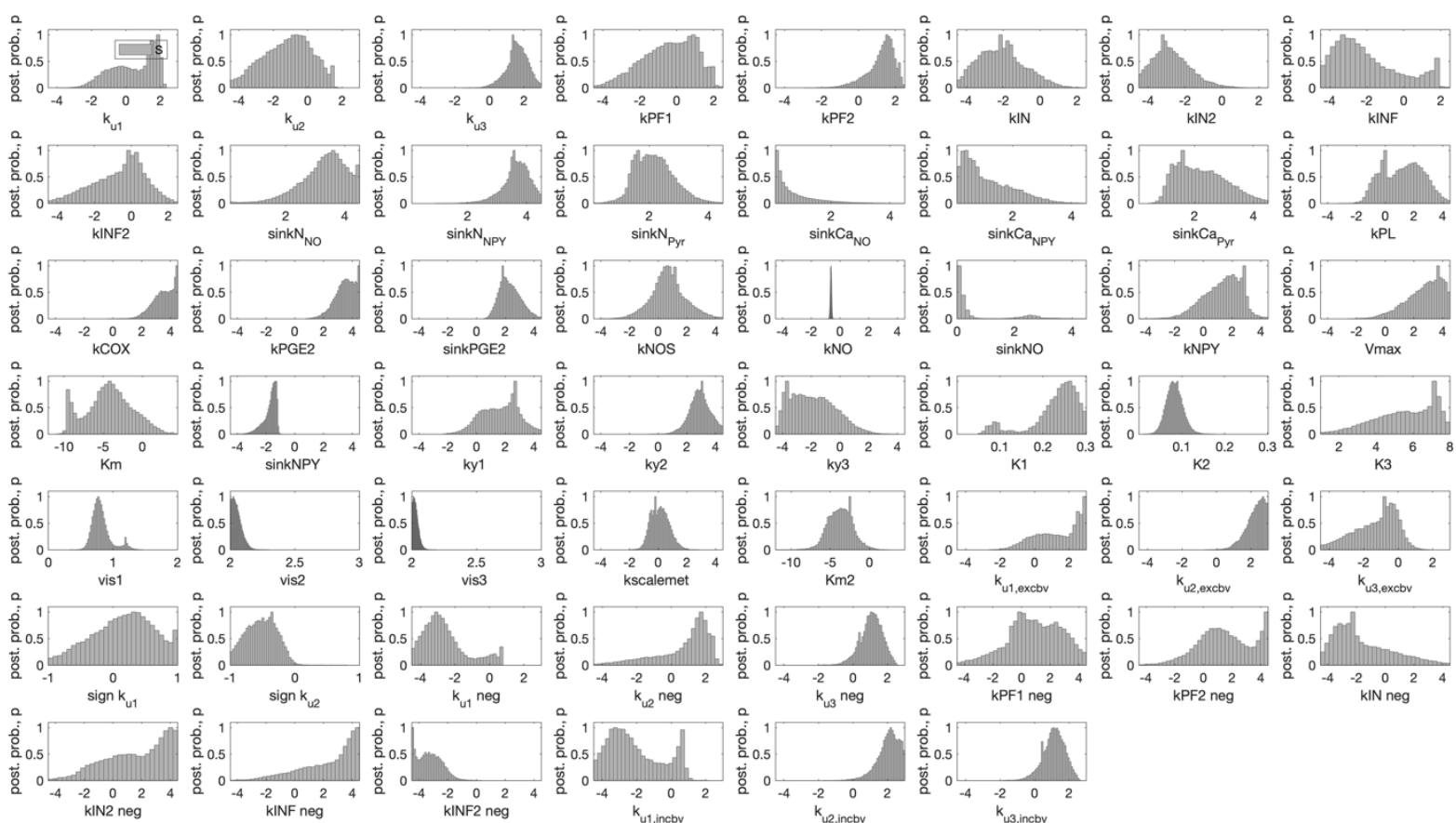

Figure S8. Posterior probability profile ( $y$-axis) for each estimated model parameter ( $x$-axis, $\log _{10}$ space) for the model estimated to data from Huber et al. [6]. The affix 'neg' represent parameter value for negative response. The affix 'excbv' and 'incbv' represent parameter values for the excitatory and inhibitory task, as presented in Figure 7BC, respectively. 


\section{References}

[1] Drew PJ, Shih AY, Kleinfeld D. Fluctuating and sensory-induced vasodynamics in rodent cortex extend arteriole capacity. Proc Natl Acad Sci U S A 2011; 108: 8473-8478.

[2] Uhlirova H, Kilic K, Tian P, et al. Cell type specificity of neurovascular coupling in cerebral cortex. Elife; 5. Epub ahead of print 2016. DOI: 10.7554/eLife.14315.

[3] Sten S, Elinder F, Cedersund G, et al. A quantitative analysis of cell-specific contributions and the role of anesthetics to the neurovascular coupling. Neuroimage; 215. Epub ahead of print 2020. DOI: 10.1016/j.neuroimage.2020.116827.

[4] Desjardins M, Kılıç K, Thunemann M, et al. Awake Mouse Imaging: From Two-Photon Microscopy to Blood Oxygen Level-Dependent Functional Magnetic Resonance Imaging. Biol Psychiatry Cogn Neurosci Neuroimaging 2019; 4: 533-542.

[5] Shmuel A, Augath M, Oeltermann A, et al. Negative functional MRI response correlates with decreases in neuronal activity in monkey visual area V1. Nat Neurosci 2006; 9: 569-577.

[6] Huber L, Goense J, Kennerley AJ, et al. Investigation of the neurovascular coupling in positive and negative $\{$ BOLD $\}$ responses in human brain at 7 T. Neuroimage 2014; 97: 349-362. 